\title{
SCExAO/CHARIS Near-infrared Direct Imaging, Spectroscopy, and Forward-Modeling of $\kappa$ And b: A Likely Young, Low-gravity Superjovian Companion
}

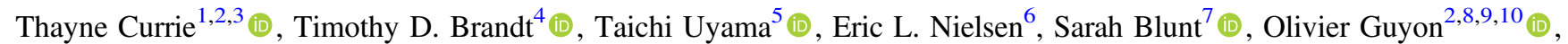
Motohide Tamura $^{5,10}$ (10), Christian Marois ${ }^{11,12}$ (1) , Kyle Mede ${ }^{5}$ (i), Masayuki Kuzuhara ${ }^{10}$, Tyler D. Groff ${ }^{13}$, Nemanja Jovanovic ${ }^{14}$, N. Jeremy Kasdin ${ }^{15}$, Julien Lozi ${ }^{2}$, Klaus Hodapp ${ }^{16}$ (1) Jeffrey Chilcote ${ }^{17}$ (i), Joseph Carson ${ }^{18}$, Frantz Martinache ${ }^{19}$ (1), Sean Goebel ${ }^{16}$ (1) , Carol Grady $^{3,13}$, Michael McElwain ${ }^{13}$, Eiji Akiyama ${ }^{20}$ (1), Ruben Asensio-Torres ${ }^{21}$ (10), Masa Hayashi ${ }^{22}$, Markus Janson $^{21}$ (1), Gillian R. Knapp ${ }^{23}$ (1) , Jungmi Kwon ${ }^{24}$ (1) , Jun Nishikawa ${ }^{22}$, Daehyeon $\mathrm{Oh}^{25}$ (1) Joshua Schlieder ${ }^{13}$ (1), Eugene Serabyn ${ }^{26}$, Michael Sitko ${ }^{27}$ (10), and Nour Skaf ${ }^{28}$

${ }^{1}$ NASA-Ames Research Center, Moffett Field, CA, USA; thayne.m.currie@nasa.gov, currie@naoj.org

${ }^{2}$ Subaru Telescope, National Astronomical Observatory of Japan, 650 North A'ohōkū Place, Hilo, HI 96720, USA

${ }^{3}$ Eureka Scientific, 2452 Delmer Street Suite 100, Oakland, CA, USA

${ }^{4}$ Department of Physics, University of California, Santa Barbara, Santa Barbara, CA, USA

${ }_{6}^{5}$ Department of Astronomy, The University of Tokyo, 7-3-1, Hongo, Bunkyo-ku, Tokyo 113-0033, Japan

${ }^{6}$ Kavli Institute for Particle Astrophysics and Cosmology, Stanford University, Stanford, CA 94305, USA

${ }^{7}$ Department of Astronomy, Harvard University, 60 Garden St., Cambridge, MA, USA

${ }^{8}$ Steward Observatory, University of Arizona, Tucson, AZ 85721, USA

${ }_{10}^{9}$ College of Optical Sciences, University of Arizona, Tucson, AZ 85721, USA

${ }^{10}$ Astrobiology Center of NINS, 2-21-1, Osawa, Mitaka, Tokyo, 181-8588, Japan

${ }^{11}$ National Research Council of Canada Herzberg, 5071 West Saanich Rd, Victoria, BC, V9E 2E7, Canada

${ }^{12}$ University of Victoria, 3800 Finnerty Rd, Victoria, BC, V8P 5C2, Canada

${ }^{13}$ NASA-Goddard Space Flight Center, Greenbelt, MD, USA

${ }^{14}$ Department of Astronomy, California Institute of Technology, 1200 E. California Blvd., Pasadena, CA 91125, USA

${ }^{15}$ Department of Mechanical Engineering, Princeton University, Princeton, NJ, USA

${ }^{16}$ Institute for Astronomy, University of Hawaii, 640 North A 'ohōkū Place, Hilo, HI 96720, USA

${ }^{17}$ Department of Physics, University of Notre Dame, 225 Nieuwland Science Hall, Notre Dame, IN 46556, USA

18 Department of Physics, College of Charleston, Charleston, SC 29424, USA

${ }^{19}$ Université Côte d'Azur, Observatoire de la Côte d'Azur, CNRS, Laboratoire Lagrange, France

${ }^{20}$ Hokkaido University, Department of Cosmosciences, Hokkaido University, Kita-ku, Sapporo, Hokkaido 060-0810, Japan

${ }^{21}$ Department of Astronomy, Stockholm University, AlbaNova University Center, SE-106 91 Stockholm, Sweden

22 National Astronomical Observatory of Japan, 2-21-2, Osawa, Mitaka, Tokyo 181-8588, Japan

${ }^{23}$ Department of Astrophysical Sciences, Princeton University, Princeton, NJ, USA

${ }^{24}$ ISAS/JAXA, 3-1-1 Yoshinodai, Chuo-ku, Sagamihara, Kanagawa 252-5210, Japan

25 National Meteorological Satellite Center, 64-18 Guam-gil, Gawnghyewon-myeon, Jincheon-gun, Chungcheongbuk-do 27803, Republic of Korea

26 Jet Propulsion Laboratory, M/S 171-113, California Institute of Technology, Pasadena, CA 91109, USA

${ }^{27}$ Center for Extrasolar Planetary Systems, Space Science Institute, 1120 Paxton Ave., Cincinnati, OH 45208, USA

${ }^{28}$ Institut d'Optique Graduate School, Université Paris-Saclay, Paris, France

Received 2018 September 18; revised 2018 October 15; accepted 2018 October 17; published 2018 November 30

\begin{abstract}
We present SCExAO/CHARIS high-contrast imaging/JHK integral field spectroscopy of $\kappa$ And b, a directly imaged low-mass companion orbiting a nearby $\mathrm{B} 9 \mathrm{~V}$ star. We detect $\kappa$ And $\mathrm{b}$ at a high signal-to-noise ratio and extract high-precision spectrophotometry using a new forward-modeling algorithm for (A-)LOCI complementary to KLIP-FM developed by Pueyo et al. $\kappa$ And b's spectrum best resembles that of a low-gravity L0-L1 dwarf $(\mathrm{L} 0-\mathrm{L} 1 \gamma)$. Its spectrum and luminosity are very well matched by 2MASS J0141-4633 and several other $12.5-15 M_{\mathrm{J}}$ free-floating members of the $40 \mathrm{Myr}$ old Tuc-Hor Association, consistent with a system age derived from recent interferometric results for the primary, a companion mass at/near the deuterium-burning limit $\left(13_{-2}^{+12} \mathrm{M}_{\mathrm{J}}\right)$, and a companion-to-primary mass ratio characteristic of other directly imaged planets $\left(q \sim 0.005_{-0.001}^{+0.005}\right)$. We did not unambiguously identify additional, more closely orbiting companions brighter and more massive than $\kappa$ And $\mathrm{b}$ down to $\rho \sim 0$ !" $3(15 \mathrm{au})$. SCExAO/CHARIS and complementary Keck/NIRC2 astrometric points reveal clockwise orbital motion. Modeling points toward a likely eccentric orbit: a subset of acceptable orbits include those that are aligned with the star's rotation axis. However, $\kappa$ And b's semimajor axis is plausibly larger than 55 au and in a region where disk instability could form massive companions. Deeper high-contrast imaging of $\kappa$ And and low-resolution spectroscopy from extreme adaptive optics systems such as SCExAO/CHARIS and higher-resolution spectroscopy from Keck/OSIRIS or, later, IRIS on the Thirty Meter Telescope could help to clarify $\kappa$ And b's chemistry and whether its spectrum provides an insight into its formation environment.
\end{abstract}

Key words: planetary systems - stars: early-type - stars: individual: (HD 222439) - techniques: high angular resolution

\section{Introduction}

In the past decade, high-contrast imaging systems and now dedicated extreme adaptive optics-based planet imagers have revealed the first direct detections of planets around nearby, young stars (Marois et al. 2008, 2010a; Lagrange et al. 2010; Carson et al. 2013; Kuzuhara et al. 2013; Quanz et al. 2013; Rameau et al. 2013; Currie et al. 2014b, 2015a; Macintosh et al. 2015; Chauvin et al. 2017; Keppler et al. 2018). Their 
range of masses $\left(2-15 M_{\mathrm{J}}\right)$ and orbital separations (10-150 au) make them key probes of models of Jovian planet formation (e.g., Boss 1997; Kenyon \& Bromley 2009; Lambrechts \& Johansen 2012). The companions' photometry reveals clear differences from field brown dwarfs and evidence for extremely cloudy and/or dusty atmospheres (Currie et al. 2011).

Integral field spectrographs (IFSs) further clarify the atmospheric properties of young planet-mass companions, revealing telltale signs of low surface gravity from sharper, more pointlike $H$-band peaks (e.g., Barman et al. 2011; Allers \& Liu 2013). Hotter, early L-type planets at very young ages (1-10 Myr) may also exhibit a red, rising slope through the $K$ band, also a sign of low surface gravity (Canty et al. 2013; Currie et al. 2014b). While the near-infrared (near-IR) spectra of some cooler L/T and T-type directly imaged planets show evidence for more extreme clouds, more vigorous chemical mixing, and/or lower gravities than found in (nearly all of) even the youngest, lowest-mass objects formed by cloud fragmentation (e.g., Currie et al. 2011; Bonnefoy et al. 2016; Rajan et al. 2017; Chauvin et al. 2018), L-type young directly imaged planets can be nearly indistinguishable from freefloating, planet-mass analogues with identical ages (e.g., Allers \& Liu 2013; Chilcote et al. 2017; Dupuy et al. 2018).

The directly imaged low-mass companion to the B9V star $\kappa$ Andromedae ( $\kappa$ And b; Carson et al. 2013) is an object whose properties could be clarified by new, high-quality IFS data. Based on $\kappa$ And b's luminosity and the primary's proposed status as a sibling of HR 8799 in the 30-40 Myr old Columba association (Zuckerman et al. 2011; Bell et al. 2015), Carson et al. estimated its mass to be $12.8 M_{\mathrm{J}}$. Using broadband photometry, Bonnefoy et al. (2014a) suggest a spectral type of M9-L3 and find some evidence for photospheric dust but fail to constrain $\kappa$ And b's surface gravity and admit a wider range of possible ages and thus masses. The Project 1640 IFS-based follow-up study by Hinkley et al. (2013) questions whether $\kappa$ And is a Columba member, derives a much older age of $220 \mathrm{Myr}$, and argues that $\kappa$ And b's spectrum suggests that the companion is not of planetary mass. However, subsequent studies based on the primary admit the possibility that the system is young ( $t \sim 30-40 \mathrm{Myr}$; and thus the companion could have low mass) (Brandt \& Huang 2015; David \& Hillenbrand 2015). Furthermore, CHARA interferometry that precisely constrains the rotation rate, gravity, temperature, and luminosity, and comparison of these properties to stellar evolution models, favors a young age (Jones et al. 2016). New, higher quality IFS data for $\kappa$ And $b$ can better clarify whether the companion shares properties (e.g., surface gravity) that are more similar to those of young planet-mass objects or older, deuterium-burning brown dwarfs.

In this study, we report new $J H K$ direct imaging and spectroscopy of $\kappa$ And b obtained with the Subaru Coronagraphic Extreme Adaptive Optics (SCExAO) project coupled to the CHARIS IFS (Groff et al. 2014; Jovanovic et al. 2015a). We analyze these data and combine them with archival Keck/NIRC2 imaging to yield new constraints on $\kappa$ And b's atmosphere and orbit.

\section{SCExAO/CHARIS Data for $\kappa$ And}

\subsection{Observations and Basic Data Reduction}

SCExAO targeted $\kappa$ And on UT 2017 September 8 with the CHARIS IFS operating in low-resolution $(R \sim 20)$, broadband
$(1.13-2.39 \mu \mathrm{m})$ mode (Peters et al. 2012; Groff et al. 2014, 2015). SCExAO/CHARIS data were acquired in pupil tracking/angular differential imaging (ADI) mode (Marois et al. 2006) with the star's light blocked by the Lyot coronagraph with the 217 mas diameter occulting spot. Satellite spots-diffractive attenuated copies of the stellar point-spread function (PSF) — were generated by applying a $25 \mathrm{~nm}$ amplitude modulation to the deformable mirror (Jovanovic et al. $2015 b$ ). Exposures consisted of 42 coadded $20.6 \mathrm{~s}$ frames covering a modest total parallactic angle rotation of $\sim 10^{\circ} .5$. The data were taken under good, "slow" seeing conditions: 0 ". 4-0." 5 in the $V$ band with $2-4 \mathrm{~m} \mathrm{~s}^{-1}$ winds. The real-time adaptive optics (AO) telemetry monitor recorded the residual wavefront error after SCExAO's correction, implying typical exposure-averaged $\mathrm{H}$-band $\mathrm{Strehl}$ ratios of $90 \%-92 \%$.

We used the CHARIS Data Reduction Pipeline (CHARIS DRP; Brandt et al. 2017) to convert raw CHARIS data into data cubes consisting 22 image slices spanning wavelengths from 1.1 to $2.4 \mu \mathrm{m}$. Calibration data provided a wavelength solution; using the the robust "least squares" method described by Brandt et al., we extracted CHARIS data cubes. Contemporaneous Keck/NIRC2 observations of HD 1160 calibrated CHARIS astrometry, yielding a spaxel scale of 0 ." 0162 , a field of view of radius $\rho \sim 1$." 05 , and north position angle offset of -2.2 (see Appendix A).

Basic image processing steps-e.g., image registration, sky subtraction-were carried out using our CHARIS IDL-based data reduction pipeline, which will later be released alongside a future release of the Python-based CHARIS DRP (i.e., the "CHARIS Post-Processing Pipeline"), and were described in recent SCExAO/CHARIS science/instrumentation studies (Currie et al. 2018; Goebel et al. 2018). Inspection of the data cubes revealed little residual atmospheric dispersion and exposure-to-exposure motion of the centroid position; the spot modulation amplitude translated into a channel-dependent spot extinction of atten $_{\lambda}=2.72 \times 10^{-3} \times(\lambda / 1.55 \mu \mathrm{m})^{-2}$.

To spectrophotometrically calibrate each data cube, we considered both stellar atmosphere models and the widely used library of Pickles (1998) adopted in our previous CHARIS papers (Currie et al. 2018; Goebel et al. 2018), in the GPI Data Reduction Pipeline (Perrin et al. 2014), and in P1640 analysis of $\kappa$ And b's near-IR spectrum in Hinkley et al. (2013). As described in the Appendix B, for B9V and some other spectral types the library of Pickles lacks direct measurements in the near-IR and instead adopts an extrapolation from shorter wavelengths that would translate into a miscalibrated companion spectrum. As an alternative, we used a Kurucz stellar model atmosphere (Castelli \& Kurucz 2004). Parameters were tuned to closely match those determined from interferometry (Jones et al. 2016): $T_{\text {eff }}=11,000 \mathrm{~K}, \log (g)=4.0 .^{29}$

As shown in Figure 1, $\kappa$ And $b$ is visible in raw CHARIS data, with a peak emission roughly three times (0.5-5 times) that of the local speckle intensity in wavelength-collapsed images (individual channels). In the $H$ band, the companion is about as well separated from the speckle halo as it was in earlier SCExAO/HiCIAO data from Fall 2016 obtained with the vortex coronagraph shown in Figure 6(a) of Kuhn et al. (2018). Inspection of our raw broadband images shows that $\kappa$

\footnotetext{
29 The nearest model of Pickles with complete near-IR coverage (A0V) or Kurucz models at slightly different temperatures/gravities (e.g., $T_{\text {eff }}=10,500 \mathrm{~K}$, $\log (g)=4.5)$ yielded an identical calibration to within $\sim 2 \%$ across the CHARIS bandpass.
} 


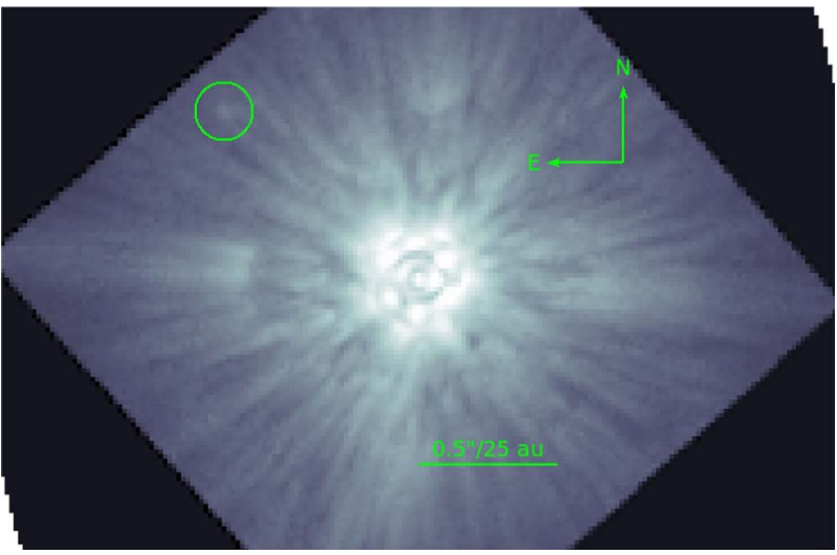

Figure 1. A characteristic broadband (wavelength-collapsed) CHARIS image shown in a log color stretch (minimum value to maximum value). The companion $\kappa$ And $\mathrm{b}$ is visible without any PSF subtraction techniques or even unsharp masking applied. The stellar halo is well suppressed at an intensity roughly equal to or just slightly higher than that of $\kappa$ And $\mathrm{b}$ down to $\rho \approx 0$." 5 .

And $\mathrm{b}$ would be marginally visible without processing at smaller separations down to $\rho \sim 0$ ". 5 .

\subsection{PSF Subtraction and Spectral Extraction}

To further suppress the stellar halo and yield a detection of $\kappa$ And $b$ with a high signal-to-noise ratio $(\mathrm{S} / \mathrm{N})$ in each channel, we employed advanced PSF subtraction techniques. We performed PSF subtraction using Adaptive, Locally Optimized Combination of Images (A-LOCI Currie et al. 2012) - a derivative of the LOCI algorithm (Lafrenière et al. 2007). ${ }^{30}$ In this approach, the PSF $I$ of image slice $i$ in an annular region $s$ is subtracted from a weighted linear combination of other image slice regions $\{j\}$ in the sequence

$$
\mathcal{R}_{i, s}=I_{i, s}-\sum_{j} \alpha_{i j, s} I_{j, s}
$$

In LOCI, the coefficients $\alpha_{i j, s}$ are determined by solving a system of linear equations that minimize the residuals between the target slice and references in an "optimization" region $o$, the solution to the linear system

$$
\boldsymbol{A} \cdot \boldsymbol{\alpha}=\boldsymbol{b},
$$

where the covariance matrix $\boldsymbol{A}$ and column matrix $\boldsymbol{b}$ are

$$
\mathrm{A}_{j l}=\sum_{\text {pixels } k} I_{j k, o} I_{l k, o} \quad \text { and } \quad \mathrm{b}_{j}=\sum_{\text {pixels } k} I_{i k, o} I_{j k, o},
$$

by a simple matrix inversion. The subtraction zone $s$ is typically a subset of pixels comprising optimization region $o$. The set of image slices $J$ used to construct a weighted reference PSF is typically defined by those fulfilling a rotational gap criterion, where a point source in region $s$ has moved some

\footnotetext{
${ }^{30}$ We did not use the Karhunen-Loève image projection (KLIP) algorithm (Soummer et al. 2012). At full rank (i.e. directly inverting the full covariance matrix), (A-)LOCI and KLIP use different formalisms but they are mathematically equivalent; using SVD to compute the pseudo-inverse of the covariance matrix in (A-)LOCI is similar to truncating the basis set in KLIP (Marois et al. 2010b; Currie et al. 2014a, 2014c; Savransky 2015). In previous direct comparisons, A-LOCI tended to yield detections with higher S/N (up to a factor of 2-3) (e.g., Rameau et al. 2013) and more whitened residual noise. However, in practice, the algorithms simply differ in setup: in whether they use optimization/training zones to construct a PSF model removed from a smaller subtraction zone, perform masking, and/or use correlation-based frame selection.
}

fraction of a PSF footprint, $\delta \times \theta_{\mathrm{FWHM}}$, between frames $i$ and $j$ due to parallactic angle motion.

In A-LOCI, this approach is modified in several ways. First, it optionally removes pixels within the subtraction zone $s$ from the optimization zone $o$, which increase point-source throughput and-as shown in Appendix C-makes algorithm forwardmodeling more tractable ("local masking"/"a moving pixel mask"; Marois et al. 2010b; Currie et al. 2012, 2015b). Second, it redefines the covariance matrix $\boldsymbol{A}$ and column matrix $\boldsymbol{b}$, selecting the $n$ image slices that are best correlated with the target image slice over each region $o$. Third, it rewrites $\boldsymbol{A}$ using singular value decomposition (SVD) as $\boldsymbol{U} \boldsymbol{\Sigma} \boldsymbol{V}$, truncating the diagonal matrix, $\Sigma$, at singular values greater than some fraction of the maximum singular value $\left(s v d_{\mathrm{lim}}\right)$ before inverting and thus allowing a low(er)-rank approximation of the covariance matrix $\boldsymbol{A}$ :

$$
\boldsymbol{\alpha}=\left(\boldsymbol{U} \boldsymbol{\Sigma}_{>s v d_{\lim }} \boldsymbol{V}\right)^{-1} \cdot \boldsymbol{b} .
$$

We performed two reductions: (1) a conservative one focused on obtaining a high-fidelity spectrum and (2) an aggressive one that maximizes the achieved contrast in our data. In our first, "conservative" approach, we processed data in annular regions for each wavelength channel independently (ADI only). The annular subtraction zone of depth $d r=10$ was masked, a weighted reference PSF was constructed from a 75 PSF footprint "optimization" area exterior to the subtraction zone, and the diagonal terms of the covariance matrix were truncated at $s v d_{\text {lim }}=2 \times 10^{-6} \times \max (\Sigma)$. In our second, "aggressive" approach, we performed an A-LOCI reduction first, utilizing ADI only, and then performed spectral differential imaging (SDI) on the ADI residuals. For the ADI component, we shrunk the rotation gap, optimization area, and SVD cutoff, leaving unmasked the subtraction zone, which was $d r=5$ pixels deep . For the SDI component, we scaled each image slice in the ADIreduced data cube by wavelength and subtracted the residuals with A-LOCI. Instead of an angular gap, we imposed a radial gap of $\delta=0.65$, masked the subtraction zone, and constructed a weighted reference PSF from pixels at the same separation as the subtraction zone but different angles as in Currie et al. (2017b) from the pseudo-inverse of $\boldsymbol{A}$ truncated at $s v d_{\mathrm{lim}}=$ $1 \times 10^{-6} \times \max (\Sigma)$. In all cases, given the limited number of exposures, we did not truncate the reference set by crosscorrelation. Finally, we descaled, rotated, and combined the ADI/SDI-subtracted image slices together for a final data cube and final broadband (wavelength-collapsed) image.

To assess and correct for signal loss of $\kappa$ And $b$ due to processing and thus extract a calibrated spectrum and precise astrometry, we forward-modeled planet spectra through the observing sequence (e.g., Pueyo 2016). Our formalism extends that of Brandt et al. (2013), is detailed in Appendix C, and considers both self-subtraction due to displaced copies of the planet signal weighted by coefficients $\alpha_{i j}$ and perturbations of these coefficients $\beta_{i j}$ due to the planet signal.

\subsection{Detection at High $S / N$ of $\kappa$ And $b$ with SCExAO/CHARIS and Extracted Spectrum}

Figures 2 and 3 display wavelength-collapsed CHARIS images reduced using "conservative" and "aggressive" PSF subtraction approaches and utilizing ADI only and in 

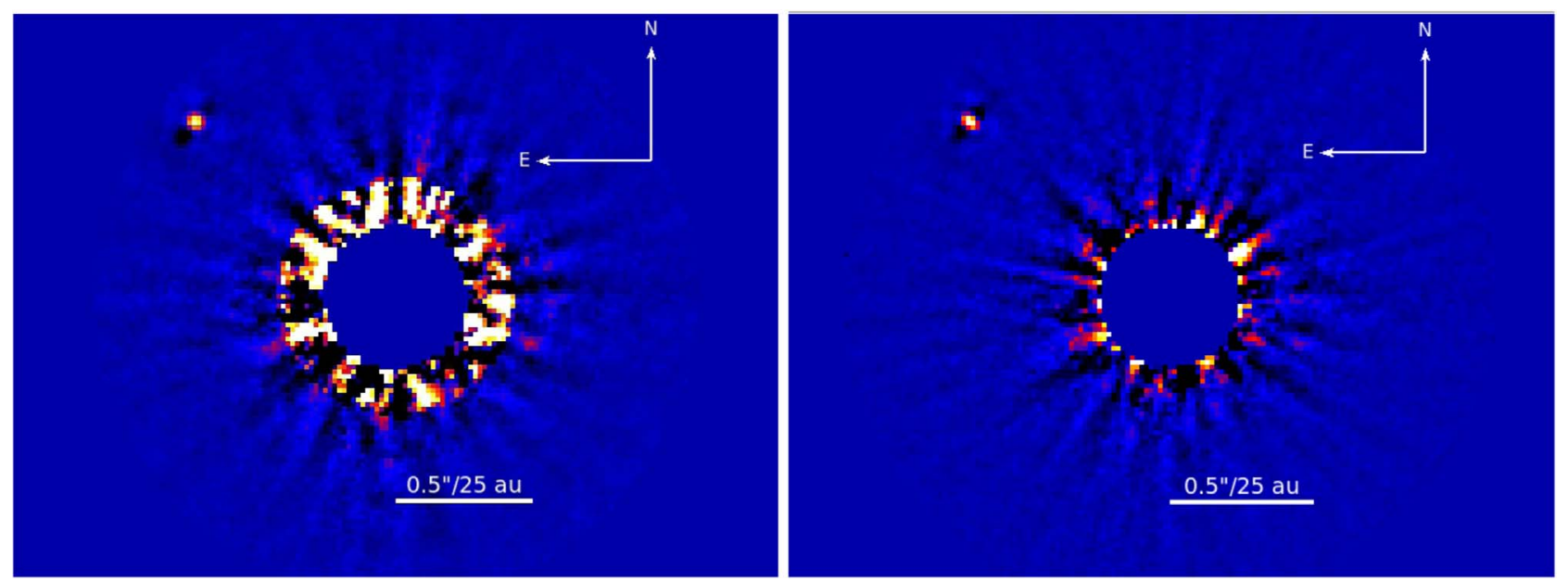

Figure 2. Detection of $\kappa$ And $b$ from SCExAO/CHARIS utilizing only ADI (not SDI) (left) with very conservative settings in A-LOCI for PSF subtraction and (right) with a more aggressive reduction using A-LOCI. $\kappa$ And b is detected with S/N of 88 and 110 as defined in Mawet et al. (2014). We extract the spectrum of $\kappa$ And $\mathrm{b}$ from the conservative reduction. The residuals are significantly higher at $\rho \sim 0$ ! $3-0 . " 5$ for the conservative reduction, largely due to a combination of local masking, aggressive covariance matrix truncation, and the large rotation gap. Throughput is lower and self-subtraction footprints along the azimuthal direction are stronger for the aggressive reduction due to its lack of masking, its less aggressive covariance matrix truncation, and smaller rotation gap.

conjunction with SDI. The companion $\kappa$ And $\mathrm{b}$ is easily visible at a high $\mathrm{S} / \mathrm{N}(88-210)$ in the wavelength-collapsed images at a projected separation of $\rho \approx 0$ ".91 and decisively detected in all channels in all reductions. Except for channel 6 in the most conservative reduction $\left(\lambda_{\mathrm{o}}=1.376 \mu \mathrm{m} ; \mathrm{S} / \mathrm{N} \sim 6.4\right)$, the detection significance exceeds $10 \sigma$ in all channels for all reductions.

To extract the spectrum for $\kappa$ And $\mathrm{b}$ from the conservative (ADI only) reduction, we defined the signal from aperture photometry with $r_{\text {ap }}=0.5 \lambda / D$ around the best-estimated position (as determined from the wavelength-collapsed image). We repeated these steps with slight modifications to our algorithm settings to confirm repeatability of the spectrum to a level less than the intrinsic $\mathrm{S} / \mathrm{N}$ of the detection in each channel. We confirmed that a negative copy of the extracted planet spectrum, when inserted into our sequence prior to processing, fully nulled $\kappa$ And $\mathrm{b}$ in all channels after PSF subtraction.

Figure 4 displays the extracted CHARIS spectrum in units of mJy (left) and (right) compares our spectrum to that from P1640 as extracted in Hinkley et al. (2013) in units of erg s $\mathrm{scm}^{-1} \AA^{-1}$. The spectrum is fully listed in Appendix D. The CHARIS spectrum shows regions of suppressed flux in between the $J H K$ passbands and a slight suppression beyond $2.3 \mu \mathrm{m}$, attributed to water and water/CO absorption in early L dwarfs (e.g., Cushing et al. 2005, 2008; Cruz et al. 2018). The $H$-band spectrum is characterized by a clear peak at $\lambda \sim 1.65 \mu \mathrm{m}$ and steep drop at redder wavelengths; the $K$-band spectrum exhibits a plateau or slightly rising flux between 2.1 and $2.2 \mu \mathrm{m}$.

The CHARIS spectrum shows slight differences from that extracted from P1640 over wavelengths where the two overlap $(1.1-1.8 \mu \mathrm{m})$. The CHARIS spectrum is more peaked in the $H$ band than in the P1640 data at $\sim 1.65 \mu \mathrm{m}$, with significantly lower flux density at $1.7-1.8 \mu \mathrm{m}$. Section 7.1 discusses the sources of these differences.

Following Greco \& Brandt (2016), we assess the nature of residual noise affecting our extracted spectrum by estimating the spectral covariance at $\kappa$ And b's location in our final data cube. We divided each channel by the residual noise profile and then computed the cross-correlation between pairs of channels $i$ and $j$ in a $2 \lambda / D$ wide annulus at $\kappa$ And b's location, masking

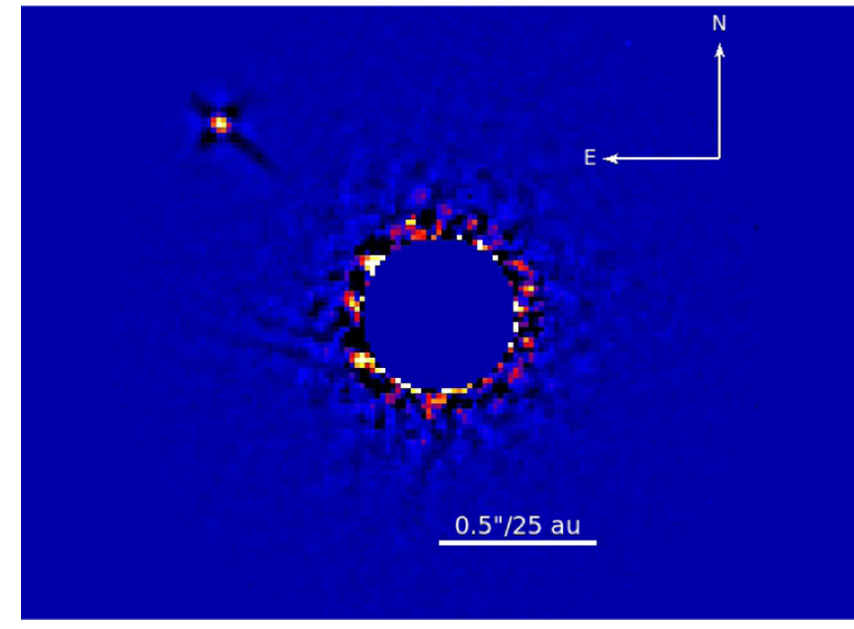

Figure 3. Detection of $\kappa$ And $b$ utilizing both ADI and SDI $(S / N \sim 210)$. Although the planet now exhibits strong radial self-subtraction footprints due to SDI, its signal loss due to SDI is nearly negligible due to CHARIS's wide spectral bandpass.

pixels within $2 \lambda / D$ of the companion:

$$
\psi_{i, j}=\frac{\left\langle\overline{C_{i} C_{j}}\right\rangle}{\sqrt{\left\langle\overline{C_{i}^{2}}\right\rangle\left\langle\overline{C_{j}^{2}}\right\rangle}} .
$$

Figure 5 displays the spectral covariance at the location of $\kappa$ And b. Except for a few red channels (e.g., 16 and 17 in the $K$ band), the covariance drops for sharply off-diagonal elements. The functional form for the covariance proposed by Greco \& Brandt (2016) consists of spatially $(\rho)$ and spectrally $(\lambda)$ correlated noise with characteristic lengths $\left(\sigma_{\rho}\right.$ and $\left.\sigma_{\lambda}\right)$ and an uncorrelated term $A_{\delta}$ :

$$
\psi_{i, j}=A_{\rho} e^{-0.5\left(\left(\lambda_{i}-\lambda_{j}\right) / \sigma_{\rho}\right)^{2}}+A_{\lambda} e^{-0.5\left(\left(\lambda_{i}-\lambda_{j}\right) / \sigma_{\lambda}\right)^{2}}+A_{\delta} .
$$

The data are best fit by $A_{\rho}=0.12, A_{\lambda}=0.05, A_{\delta}=0.82$, $\sigma_{\rho}=0.65$, and $\sigma_{\lambda}=0.24$ : thus, the residual speckle noise is well suppressed and poorly coupled between different wavelengths. At smaller separations where the rotation gap criterion results in far poorer speckle suppression, the noise is 

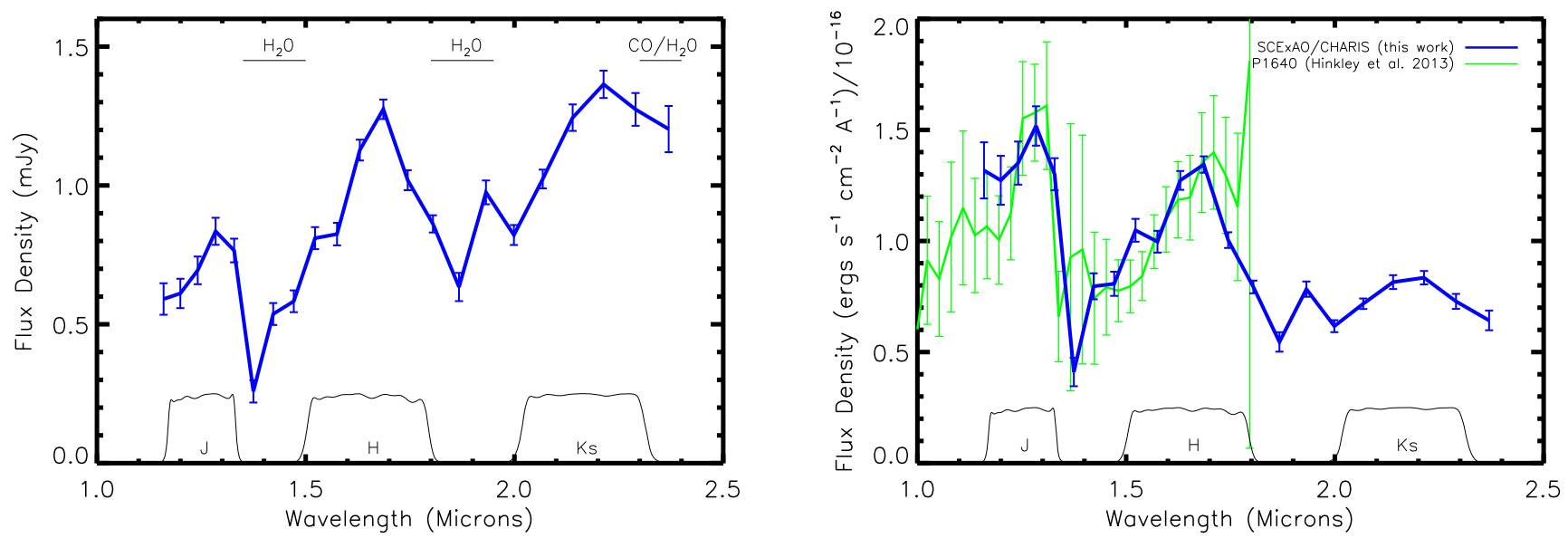

Figure 4. Left: SCExAO/CHARIS spectrum of $\kappa$ And b extracted from our conservative (ADI only) reduction shown in $F_{\nu}$ units with regions attributed major molecular absorption in substellar objects overplotted. Right: SCExAO/CHARIS spectrum of $\kappa$ And b compared to that from P1640 presented in Hinkley et al. (2013) and plotted in $F_{\lambda}$ units. Both panels show transmission profiles for major near-IR passbands $J H K_{\mathrm{S}}(\mathrm{MKO})$. The CHARIS error bars do not include an additional $\sim 5 \%$ absolute calibration uncertainty.

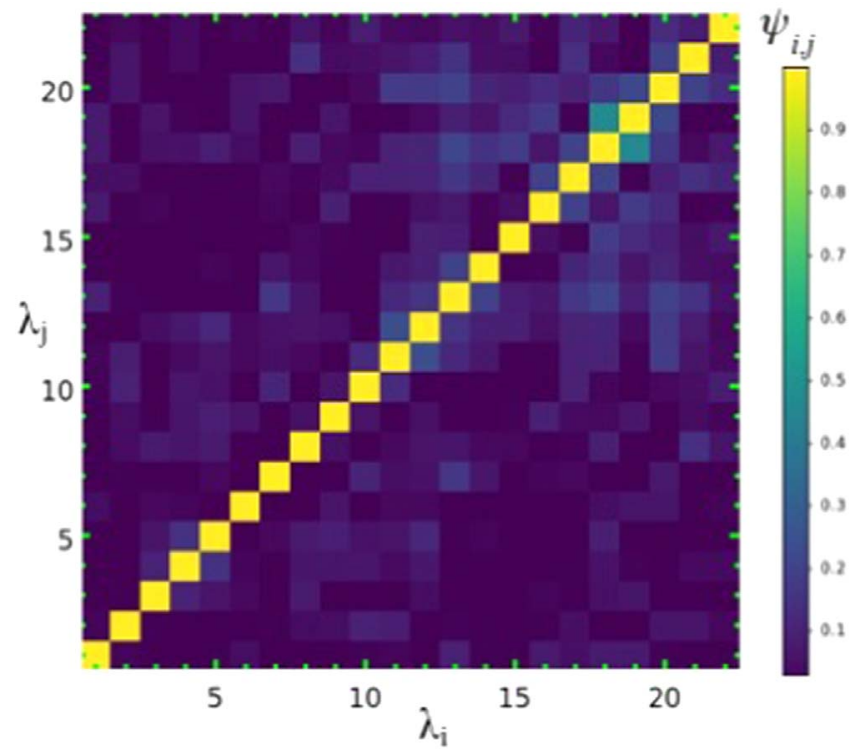

Figure 5. The correlation matrix $\psi_{i, j}$ as a function of spectral channel. Offdiagonal elements identify the effect of residual correlated noise. With the exception of a few channels (e.g., a slight coupling of channels 17 and 18), the residuals are nearly spatially uncorrelated.

dominated by the correlated components (e.g., at $\rho \sim 0$ !" 45, $A_{\rho}+A_{\lambda}=0.56$ and $\left.A_{\delta}=0.44\right)$.

To estimate broadband photometry for $\kappa$ And b, we convolve the spectrum with the Maunakea Observatories (MKO) $J H K$ filter functions binned down to the resolution of CHARIS. The companion's apparent magnitude in major MKO passbands is $J=15.84 \pm 0.09, H=15.01 \pm 0.07$, and $K_{\mathrm{s}}=$ $14.37 \pm 0.07$. Its $J-H$ and $J-K_{\mathrm{s}}$ colors agree with previous estimates from Carson et al. (2013), Hinkley et al. (2013), and Bonnefoy et al. (2014a). In the photometric system of the Two Micron All Sky Survey (2MASS), its colors are slightly redder (e.g., $J_{2 \mathrm{MASS}}-K_{\mathrm{s}, 2 \mathrm{MASS}}=1.52$ ).

\section{New and Archival Keck/NIRC2 $K_{\mathrm{s}}$-band Astrometric Data}

To supplement $\kappa$ And b's astrometry derived from SCExAO/ CHARIS data, we measure its position in well-calibrated data obtained recently and in prior epochs using Keck coupled with the NIRC2 camera. First, we obtained new Keck/NIRC2 coronagraphic imaging of $\kappa$ And on UT 2017 December 8 in the $K_{\mathrm{s}}$ filter using the 0".6 diameter coronagraphic spot. Data consisted of coadded $30 \mathrm{~s}$ exposures covering 13.6 of parallactic angle motion. Basic image processing follows previous methods utilized for observations of $\kappa$ And taken with Keck/NIRC2 drawn from Currie et al. (2011), including dark subtraction, flatfielding, distortion corrections, and image registration (Bonnefoy et al. 2014a). We used A-LOCI with local masking of the subtraction zone to produce a nearly unattenuated detection of $\kappa$ And $\mathrm{b}(\mathrm{S} / \mathrm{N}=27)$.

Second, we searched for and identified $\kappa$ And $K_{\mathrm{s}}$-band data from the Keck Observatory Archive taken on UT 2013 August 18 (PI John Asher Johnson), consisting of 15 exposures of $20 \mathrm{~s}$ each. A visual inspection of these data reveals $\kappa$ And $\mathrm{b}$, and they fill in the gap in astrometric measurements between the CHARIS data set (2017 September) and those from Bonnefoy et al. (2014a). We use A-LOCI with local masking and a rotation gap of one PSF footprint to subtract the stellar halo, yielding a high-throughput detection and high-precision astrometry. The $\mathrm{S} / \mathrm{N}$ is comparable to or slightly higher than that from the discovery paper (Carson et al. 2013) and other early detections (e.g., Burress et al. 2013). Third, we report unpublished astrometry for $\kappa$ And $\mathrm{b}$ from data taken on UT 2012 November 3 and published in Bonnefoy et al. (2014a).

The 2017 and 2013 epoch detections are shown in Figure 6. Astrometry in each data set assumed a pixel scale of 9.971 mas and north position angle offset of 0.262 for the 2017 data and a pixel scale of 9.952 mas and north position angle offset of 0.252 for earlier data sets (Service et al. 2016; Yelda et al. 2010). Comparing the position of $\kappa$ And $b$ in these two data sets and with CHARIS clearly shows that the companion's projected separation is decreasing with time.

\section{Empirical Constraints on $\kappa$ And b's Atmospheric Properties}

To analyze $\kappa$ And b's spectrum, we adopt a three-pronged approach: (1) comparing it to optically anchored L-dwarf spectral templates covering a range of gravities, (2) comparing it to a large library of empirical $J H K$ spectra for MLT dwarfs, and (3) assessing gravity from spectral indices. The templates 

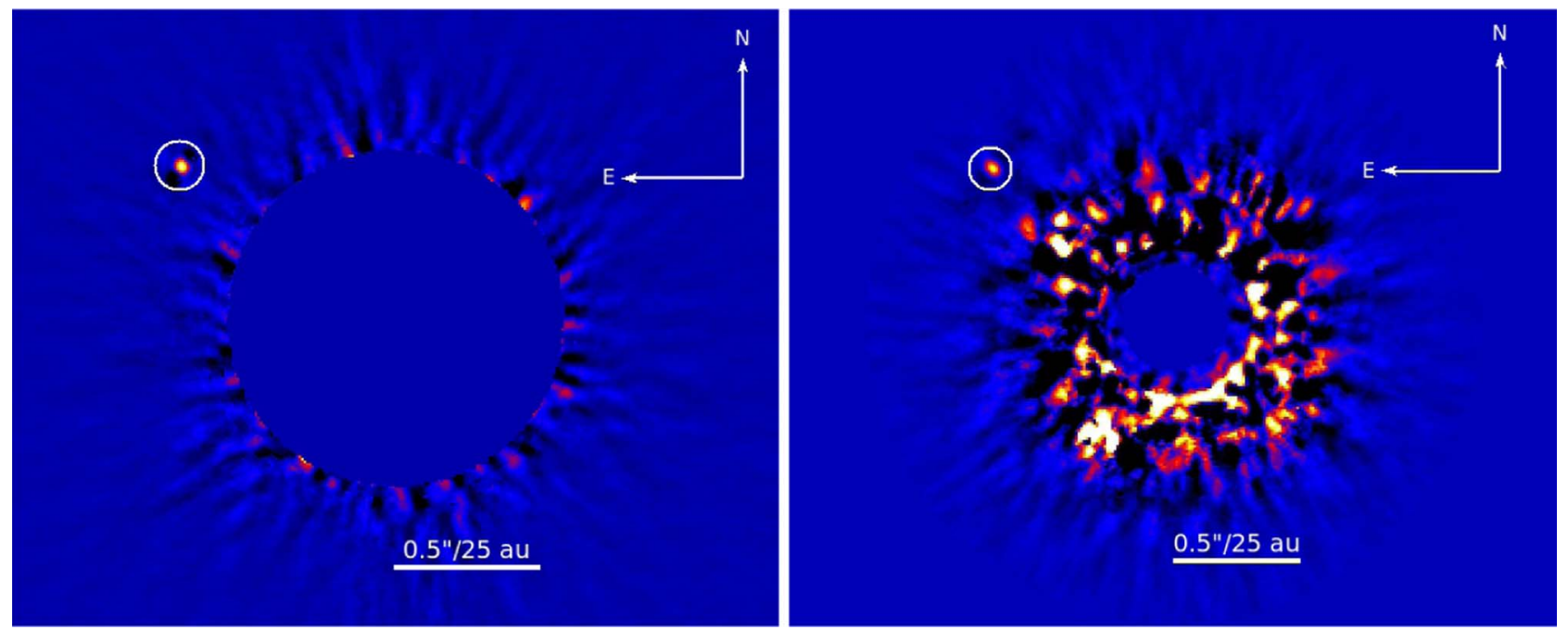

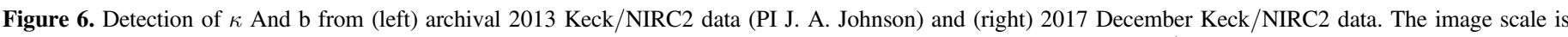
equivalent to that in previous figures; $\kappa$ And $\mathrm{b}$ is at a wider angular separation in the archival data than in the SCExAO/CHARIS data

provide a baseline qualitative assessment for $\kappa$ And b's spectral type and gravity. The libraries further clarify these parameters, identifying a set of best-fit objects, some of which have wellestimated ages and masses. The spectral indices serve as a quantitative estimate of gravity.

For empirical comparisons, we quantify the goodness-of-fit by comparing $\kappa$ And b's spectrum $f$ to the $k$ th weighted comparison spectrum $F_{k}$, choosing the multiplicative factor $\alpha_{k}$ that minimizes $\chi^{2}$ and considering errors in both $\kappa$ And $\mathrm{b}$ and the comparison spectrum:

$$
\chi_{k}^{2}=\left(f-\alpha F_{k}\right)^{T} C_{k}^{-1}\left(f-\alpha F_{k}\right) .
$$

Here, $C_{k}$ is the covariance matrix, where diagonal terms correspond to both measured errors in $\kappa$ And $\mathrm{b}\left(\sigma_{f}\right)$ and those estimated for the comparison spectrum $\left(\sigma_{F_{k}}\right)$, and off-diagonal terms consider the coupling of spectral errors in $\kappa$ And $b$ between different channels as parameterized in Section 2.3. ${ }^{31}$

We define acceptably fitting models as those with a $\chi^{2}$ per degree of freedom less than the $95 \%$ confidence limit: $\chi_{\nu}^{2} \leqslant \chi_{\nu, 95 \% \text { C.L. }}^{2}{ }^{32}$ To avoid regions heavily contaminated by tellurics and/or covering wavelengths with missing data, we primarily focused on a set of 16 CHARIS spectral channels covering the MKO JHK bandpasses. In a second pass, we focus on 11 spectral channels covering $H$ and $K$ only, where broadband spectral features may be diagnostic of gravity (Allers \& Liu 2013; Canty et al. 2013). In Chilcote et al. (2017), this is referred to as the "restricted fit." Finally, as a check on our results, for empirical comparisons we perform an

\footnotetext{
31 The spectrophotometric errors for many library spectra are non-negligible and must be considered by calculating the goodness-of-fit. Similarly, the template spectra from Cruz et al. (2018) are drawn from a collection of different sources, and thus the "template" for a given spectral type should have some uncertainty in each channel. Thus, the covariance matrix must be recomputed each time a weighted comparison spectrum is fit.

32 Greco \& Brandt (2016) discuss the effect of spectral covariance in defining the family of best-fitting solutions quantified by the $\Delta^{2}$ criterion and the $95 \%$ confidence interval about the minimum value, and note that the actual $\chi^{2}$ values including covariance can be larger. As the spectral covariance is low in our case, the diagonal terms dominate and there is only a small difference in $\chi^{2}$ including/not including the covariance. An analysis adopting a $\Delta^{2}$ instead of $\chi_{\nu}^{2}$ criterion would accept more template and empirical spectra but does not otherwise change our key results about what spectral type $\kappa$ And b best resembles.
}

"unrestricted" fit of the full $J H K$ spectrum, allowing the scaling to vary freely between the three passbands, to account for the intrinsic variation in the $J-K$ spectral energy distribution at a given spectral type (e.g., Knapp et al. 2004).

\subsection{Comparisons to Template L-dwarf Spectra}

Cruz et al. (2018) compute L-dwarf near-infrared spectral average templates, constructed (for each spectral type) from a set of characteristic optically spectral-typed substellar objects. The templates cover L0-L4 and L6-L8 field objects, L0-L1 intermediate-gravity dwarfs $(\mathrm{L} 0-\mathrm{L} 1 \beta)$, and low-gravity L0-L4 dwarfs (L0-L4 $\gamma)$. The samples of near-infrared spectra comprising each template show typical variations of the order of $\sim 5 \%$ across $J-K$; inspection of empirical spectra comprising some templates showed variations in spectral shape at similar levels. Thus, we set a floor to the spectrophotometric uncertainty of $5 \%$.

Table 1 and Figure 7 compare how well $\kappa$ And b matches each template of Cruz et al. Overall, the $\mathrm{L} 0 \gamma$ template best fits $\kappa$ And b's spectrum $\left(\chi_{\nu}^{2}=1.22\right)$, while the L3 field dwarf template fits marginally and the L0-L1 $\beta$ templates are marginally inconsistent at the $95 \%$ confidence limit. When focused more on gravitysensitive $H$ - and $K$-band, low-gravity templates $\mathrm{L} 0 \gamma$ and $\mathrm{L} 1 \gamma$ fit the best; the $\mathrm{L} 1 \beta$ and L 3 field templates are marginally consistent while the L $3 \gamma$ and L4 field templates are marginally excluded. The agreement with the overall shape of $\kappa$ And b's spectrum drives the small $\chi^{2}$ values for the L0 $\gamma$ and L3 field templates; the shape of both the $H$ - and $K$-band spectra are clearly better fit by the $\mathrm{L} 0 \gamma$ template.

\subsection{Comparisons to Empirical MLT Dwarf Spectra}

Our sample of empirical spectra primarily draws from the Montreal Spectral Library ${ }^{33}$ and from the VLT spectral library of Bonnefoy et al. (2014b). ${ }^{34}$ The Montreal library covers MLT dwarfs with field, intermediate $(\beta)$, low $(\gamma)$, and very low $(\delta)$ gravities characteristic of old $(\gtrsim 1 \mathrm{Gyr})$, intermediate aged $(\sim 100 \mathrm{Myr})$, young $\quad(\sim 10-100 \mathrm{Myr}), \quad$ and very young

\footnotetext{
33 https://jgagneastro.wordpress.com/the-montreal-spectral-library/

34 http://ipag.obs.ujf-grenoble.fr/ chauving/online_library_Bonnefoy 13. tar.gz
} 


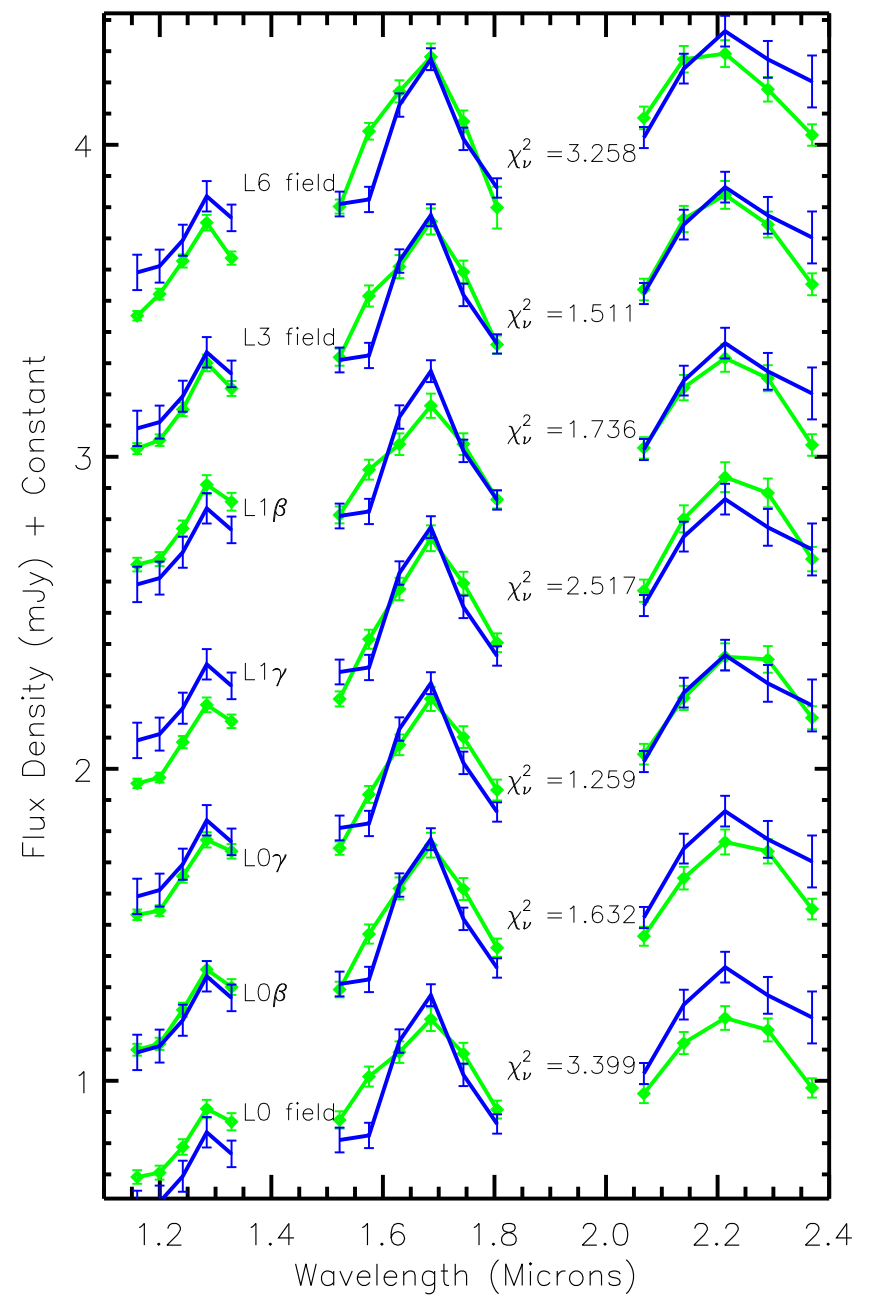

Figure 7. Comparisons between $\kappa$ And b (blue) and spectral templates (green) from Cruz et al. (2018). The wavelengths plotted are the 16 used to define $\chi^{2}$. The $\mathrm{L} 0 \gamma$ template provides the best fit; $\mathrm{L} 0 \gamma$ and $\mathrm{L} 1 \gamma$ best reproduce the shape of the $H$ - and $K$-band portions of the spectra.

Table 1

Fits to Spectral Standards of Cruz et al. (2018)

\begin{tabular}{|c|c|c|c|c|c|}
\hline $\begin{array}{l}\text { Spectral } \\
\text { Type }\end{array}$ & $\begin{array}{c}\text { Gravity } \\
\text { Class }\end{array}$ & $H_{\text {cont,CHARIS }}$ & $\begin{array}{c}\mathrm{H}_{2} \mathrm{~K}, \\
\text { CHARIS }\end{array}$ & $\chi_{\nu}^{2}($ total $)$ & $\begin{array}{r}\chi_{\nu}^{2}(H \\
+K)\end{array}$ \\
\hline L0 & field & 0.935 & 1.050 & 3.76 & 3.72 \\
\hline L0 & $\beta$ & 0.945 & 1.032 & 1.68 & 2.41 \\
\hline LO & $\gamma$ & 0.971 & 1.020 & 1.26 & 1.40 \\
\hline L1 & field & 0.912 & 1.056 & 2.90 & 3.55 \\
\hline L1 & $\beta$ & 0.926 & 1.056 & 1.80 & 1.71 \\
\hline L1 & $\gamma$ & 0.949 & 1.037 & 2.84 & 1.41 \\
\hline L2 & field & 0.896 & 1.076 & 2.03 & 2.44 \\
\hline L2 & $\gamma$ & 0.960 & 1.009 & 5.10 & 3.32 \\
\hline L3 & field & 0.890 & 1.075 & 1.51 & 1.78 \\
\hline L3 & $\gamma$ & 0.947 & 1.031 & 3.50 & 1.91 \\
\hline L4 & field & 0.867 & 1.075 & 2.28 & 1.86 \\
\hline L4 & $\gamma$ & 0.940 & 1.037 & 15.32 & 10.96 \\
\hline L6 & field & 0.847 & 1.110 & 3.36 & 2.94 \\
\hline L7 & field & 0.855 & 1.109 & 5.92 & 3.19 \\
\hline L8 & field & 0.794 & 1.172 & 5.00 & 6.63 \\
\hline
\end{tabular}

Note. The $\chi_{\nu}^{2}$ values are calculated assuming 15 degrees of freedom for fitting of the $J H K$ peaks and 10 for just $H$ and $K$. Entries in bold identify those that fit the data to within the $95 \%$ confidence limit.
$(<10 \mathrm{Myr})$ low-mass stars and substellar objects, respectively. The Montreal data draw from multiple sources presenting spectra reduced using multiple instruments, including Gagne et al. (2014, 2015), Robert et al. (2016), Aritgau et al. (2010), Delorme et al. (2012), and Naud et al. (2014). The Bonnefoy library focuses on objects near the M/L transition (M6-L1) having intermediate to (very) low gravities $(\beta \gamma \delta)$ with spectra drawn from a single source (VLT/SINFONI) reduced in a uniform manner. We trimmed our Montreal library sample of objects with very low $\mathrm{S} / \mathrm{N}$ or those with substantial telluric contamination at the edges of the $J H K$ passbands, leaving 360 objects. Since the library of Bonnefoy et al. (2014b) nominally lists a spectrum normalized in $J$ or $H K$, we focused only on those objects whose spectra can be relatively calibrated across JHK (12 objects).

Figure 8 displays $\chi_{\nu}^{2}$ as a function of spectral type for the $J H K$ and $H K$ restricted fits (top and middle panels) and the $J H K$ unrestricted fit (bottom panel), quantitatively showing how well each empirical spectrum matches $\kappa$ And b's spectrum. The distribution for the restricted fits shows a clear minimum for L0-L1 spectral types with low surface gravity with one (two) objects formally satisfying the $95 \%$ confidence limit for the full $J H K(H K)$ spectrum. In both plots, another 2-3 objects lie just above this limit, all of which are likewise L0-L1 objects with low gravity. For the unrestricted fit, more objects cluster at or below the $95 \%$ confidence limit, including the $\sim 10 \mathrm{Myr}$ old objects UScoCTIO 108B (M9.5 $\gamma$, Bonnefoy library; Bejar et al. 2008) and 2MASS J12074836-3900043 (L1 $\delta$, Montreal library; Gagne et al. 2014). The $\chi_{\nu}^{2}$ minimum for the unrestricted fit is broader (M9 to L2-L4), although L0-L1 $\gamma$ objects still dominate the subset of those that fit well.

Table 2 lists the best-fitting spectra and their properties from the restricted fits. 2MASS J0141-4633 (Kirkpatrick et al. 2006) - an $\mathrm{L} 0-\mathrm{L} 1 \gamma$ dwarf and member of the Tucana-Horologium Association - provides the best fit. ${ }^{35,36}$ In general, the sample of best-fitting objects listed in Table 2 is dominated by confirmed and candidate L0-L1 $\gamma$ Tuc-Hor members.

To illustrate how $\kappa$ And b's spectrum best resembles that of a low-gravity L0-L1 dwarf, Figure 9 compares it to 2MASS J0141-4633 (the best-fitting object with small spectrophotometric errors) and a representative set of L0-L1 objects with small errors and different gravity classes. The shape of the $H$ and $K$ spectra of $\kappa$ And $\mathrm{b}$ strongly favors that of a low-gravity object, because the $H$-band spectrum is far sharper than that of any field object and the red half of the $K$-band spectrum flatter. All other field and intermediate-gravity L0-L1 dwarfs match $\kappa$ And $\mathrm{b}$ more poorly. Other $\mathrm{L} 0-\mathrm{L} 1 \gamma$ dwarfs have $\chi^{2}$ values that are still characteristically smaller than L0-L1 field objects (see Figure 8). ${ }^{37}$

\footnotetext{
${ }^{35}$ Our adopted spectral type follows estimates from individual indices in Bonnefoy et al. (2014a) rounded to the nearest integer type.

${ }^{36}$ Considering the over 500 available spectra in the Montreal library, the L1 dwarf and candidate (10 Myr old) TW Hya member 2MASS J1148-2836 numerically provides the best fit to $\kappa$ And b's spectrum. However, like many other objects, its spectrophotometric errors are very large, and thus it was removed from our model comparisons.

${ }^{37}$ The major contributor to $\chi^{2}$ for most objects, including the L0-L1 $\gamma$ objects displayed, is the $H$-band shape, where $\kappa$ And b has a slightly sharper $H$-band shape. Some of the youngest, lowest-mass objects better match this feature (e.g., Cha 1109, UScoCTIO 108B) while more poorly matching other parts of the spectrum; a few others (e.g., KPNO Tau 4) have sharper overall $H$-band shapes.
} 

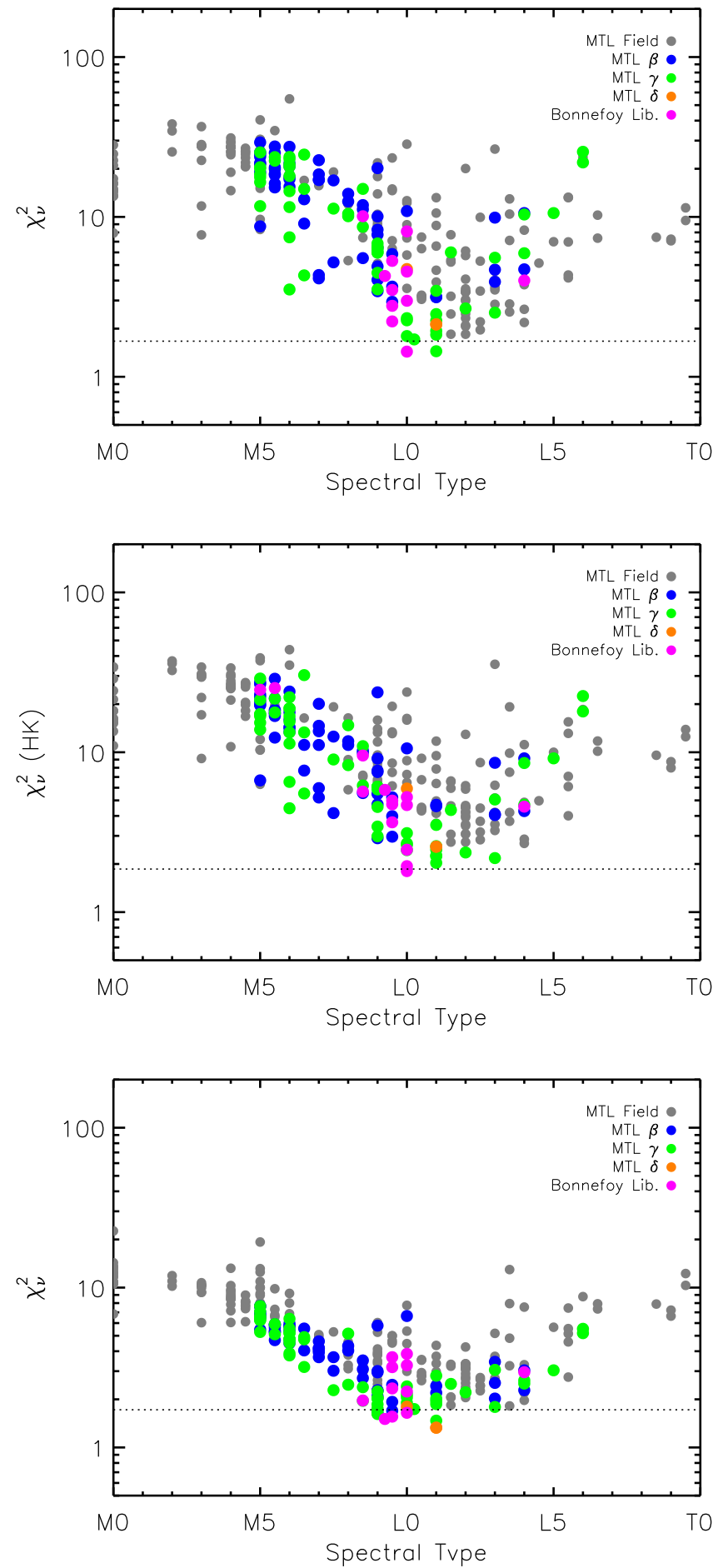

Figure 8. For the $J H K$ passbands (top) and just $H K$ (middle), the $\chi_{\nu}^{2}$ statistic comparing $\kappa$ And $\mathrm{b}$ to substellar object spectra, including objects with field (gray), intermediate (blue), low (green), and very low (orange) gravity listed in the Montreal Spectral Library and predominantly young, low-mass objects from the VLT/SINFONI library described in Bonnefoy et al. (2014a). Horizontal lines identify the $\chi_{\nu}^{2}$ limits below which objects match $\kappa$ And b's spectrum at the $95 \%$ confidence limit. Bottom: the $\chi_{\nu}^{2}$ distribution when allowing the $J-, H-$, and $K$-band portions of the empirical spectrum to be scaled separately.

\subsection{Quantitative Assessments of Surface Gravity Using Spectral Indices}

We use multiple near-infrared spectral indices to assess the companion's surface gravity: the $H$-continuum index ( $H$-cont)

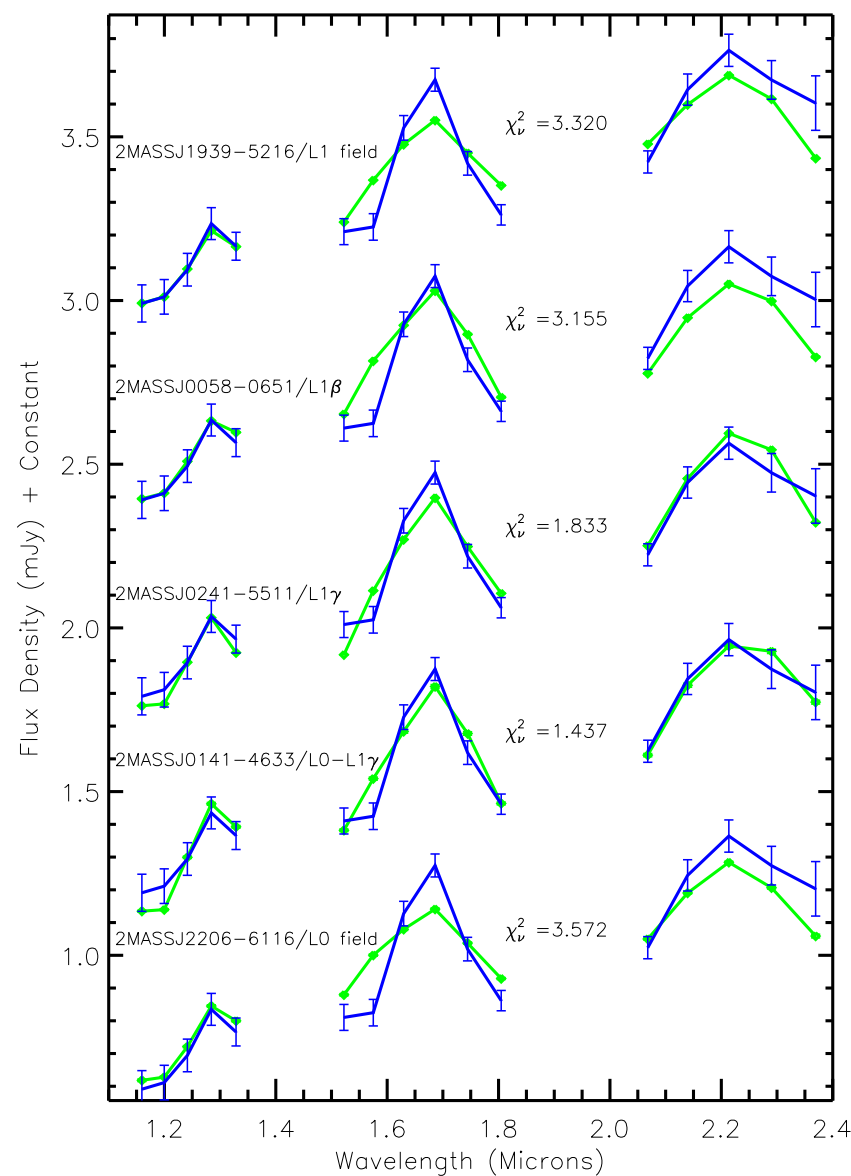

Figure 9. Comparisons between $\kappa$ And $\mathrm{b}$ (blue) and a representative sample of L0-L1 dwarfs with different gravity classes (green) from the Montreal and Bonnefoy spectral libraries. The L0-L1 $\gamma$ object 2MASS J0141-4633 provides the best overall match to $\kappa$ And $b$.

defined by Slesnick et al. (2004) and the $\mathrm{H}_{2} \mathrm{~K}$ index described by Canty et al. (2013). The $H$-cont index is defined from two measurements of the "continuum" flux $\left(\lambda_{1}=1.470 \mu \mathrm{m}, \lambda_{2}=\right.$ $1.670 \mu \mathrm{m})$ and a measurement of the "line" flux at $1.560 \mu \mathrm{m}$ :

$$
H_{\text {cont }}=\left[\frac{\lambda_{\text {line }}-\lambda_{1}}{\lambda_{2}-\lambda_{1}} F_{\lambda_{2}}+\frac{\lambda_{2}-\lambda_{\text {line }}}{\lambda_{2}-\lambda_{1}} F_{\lambda_{1}}\right] / F_{\text {line }} .
$$

The $H_{2} \mathrm{~K}$ index is defined as the flux ratio in two small bandpasses in $K: H_{2} K_{\text {ind }}=F_{\lambda, 2.17 \mu \mathrm{m}} / F_{\lambda, 2.24 \mu \mathrm{m}}$.

The wavelengths at which these spectral indices are usually evaluated do not perfectly map onto the wavelengths for each CHARIS channel in low-resolution mode, and the width of the bandpasses $(\Delta \lambda \sim 0.02 \mu \mathrm{m})$ is smaller than the change in wavelength between adjacent CHARIS channels $(\Delta \lambda \sim$ $0.05 \mu \mathrm{m})$. Thus, the spectral indices had to be modified. For $H$ cont, the change is slight: we defined the "line" flux at channel 10 $\left(\lambda_{\text {line }}=1.575 \mu \mathrm{m}\right)$ and the continuum at channels 8 and 12 $\left(\lambda_{\text {cont. }}=1.471 \mu \mathrm{m}\right.$ and $\left.1.686 \mu \mathrm{m}\right)$. Wavelengths listed by Canty et al. (2013) for the $\mathrm{H}_{2} \mathrm{~K}$ index are more poorly matched to wavelengths defining the CHARIS low-resolution channels. We therefore defined an approximate $\mathrm{H}_{2} \mathrm{~K}$ index from averages of adjacent channels 19-20 and 20-21: $H_{2} K=\left(F_{\lambda=2.139 \mu \mathrm{m}}+\right.$ $\left.F_{\lambda=2.213 \mu \mathrm{m}}\right) /\left(F_{\lambda=2.213 \mu \mathrm{m}}+F_{\lambda=2.290 \mu \mathrm{m}}\right)$.

Figure 10 compares the $H$-cont and $\mathrm{H}_{2} \mathrm{~K}$ indices for $\kappa$ And b with those from the Montreal and Bonnefoy libraries. For spectral types of M5 to L6, the typical $H$-cont indices for field 
Table 2

Properties of the Best-fitting Substellar Objects

\begin{tabular}{|c|c|c|c|c|c|c|c|c|c|c|c|}
\hline Name & $\begin{array}{c}\chi_{\nu}^{2} \\
\text { (total) }\end{array}$ & $\begin{array}{c}\chi_{\nu}^{2} \\
(H+K)\end{array}$ & $\mathrm{SpT}$ & $\begin{array}{l}H_{\text {cont. }} \\
\text { Index }\end{array}$ & $\begin{array}{c}\mathrm{H}_{2} \mathrm{~K} \\
\text { Index }\end{array}$ & Assoc. & $\begin{array}{l}\mathrm{Age} \\
(\mathrm{Myr})\end{array}$ & $\begin{array}{c}\log \left(L / L_{\odot}\right) \\
\text { (approx.) }\end{array}$ & $\begin{array}{l}T_{\text {eff }} \\
(\mathrm{K})\end{array}$ & $\begin{array}{c}\log (g) \\
\text { (approx.) }\end{array}$ & $\begin{array}{c}\text { Mass } \\
\left(M_{\mathrm{J}}\right)\end{array}$ \\
\hline 2MASS J0141-4633 & 1.43 & 1.81 & $\mathrm{~L} 0-\mathrm{L} 1 \gamma$ & 0.962 & 1.027 & Tuc-Hor & $40_{-19}^{+5}$ & -3.58 & $\begin{array}{c}1899 \pm 123 \\
1800_{-100}^{+200}\end{array}$ & $4.1-4.2$ & $13-15$ \\
\hline 2MASS J0241-5511 & 1.83 & 2.59 & $\mathrm{~L} 1 \gamma$ & 1.015 & 1.034 & Tuc-Hor & $40_{-19}^{+5}$ & -3.67 & $1731 \pm 151$ & $4.1-4.2$ & $12.5-14$ \\
\hline 2MASS J0440-5126 & 1.79 & 2.64 & $\mathrm{~L} 0 \gamma$ & 1.003 & 1.006 & Tuc-Hor? (53) & $40_{-19}^{+5} ?$ & $-3.63 ?$ & $1600-2000$ & $4.1-4.2 ?$ & $13-15 ?$ \\
\hline 2MASS J2322-6151B & 1.94 & 2.26 & $\mathrm{~L} 1 \gamma$ & 1.015 & 1.083 & Tuc-Hor & $40_{-19}^{+5}$ & -3.68 & $1793 \pm 50$ & $4.1-4.2$ & $12.5-14$ \\
\hline
\end{tabular}

Notes. Spectra for all objects match $\kappa$ And b's at $99.7 \%$ confidence for the $J H K$ restricted fit, the $H K$ restricted fit, and the $J H K$ unrestricted fit. Secure moving-group members are defined from Banyan- $\Sigma$ as those with $>95 \%$ probability in a given group. Those with $>50 \%$ are noted with "?": the Banyan- $\Sigma$ probability is listed in parentheses. Temperatures are listed from Faherty et al. (2016) (first entry) or Bonnefoy et al. (2014a) (second entry) where available; otherwise, they are estimated from the range in temperatures from Gonzales et al. (2018). If given, luminosities, surface gravities, and masses are calculated assuming the nominal object distance, the $K$-band bolometric correction from Todorov et al. (2010), and the luminosity evolution models of Baraffe et al. (2003).

${ }^{a}$ Previously identified as a Tuc-Hor member, Banyan- $\Sigma$ favors a field object ( $\sim 75 \%$ versus $\left.25 \%\right)$. No parallax is given. Thus its membership and properties depending on distance are noted with "??"

b Mass and luminosity estimated using the "optimal" kinematic distance for moving-group membership.

dwarfs range from 1 to 0.85 . Indices for young dwarfs of low/ intermediate gravity from the Montreal and Bonnefoy samples are systematically $0.05-0.10$ dex larger, exhibiting very little overlap with the field. The $\mathrm{H}_{2} \mathrm{~K}$ index appears best at selecting very young $(t<10 \mathrm{Myr})$ objects dominating the Bonnefoy sample (see also Gagne et al. 2015). The $H_{2} K$ indices for young dwarfs of low/intermediate gravity are less well separated from the field than $H$-cont indices. However, they are still characteristically smaller than for field objects, suggesting that this metric may be used to supplement an assessment of gravity derived from the $H$-cont index. Combining the two indices together retains a clear separation between nearly all young, low-gravity dwarfs and field objects. Thus, although the low resolution of CHARIS's broadband mode precludes a direct application of standard metrics for gravity in the $H$ and $K$ bands, slightly modified versions of these metrics (especially $H$-cont) can still identify likely young, low-gravity objects.

The measured gravity-sensitive indices for $\kappa$ And b$H$-cont $=1.070 \pm 0.039$ and $H_{2} K=1.055 \pm 0.041$-suggest a low surface gravity. The $H$-cont index of $\kappa$ And $\mathrm{b}$ is larger than that for any L0-L1 Montreal or Bonnefoy sample object and most similar to $H$-cont indices for $\mathrm{L} 0-\mathrm{L} 1$ objects classified as having a low gravity. The $\mathrm{H}_{2} \mathrm{~K}$ index, which is less diagnostic of surface gravity, is less conclusive since $\kappa$ And b's value overlaps with the values for both field and low-gravity objects. However, considering both indices together, $\kappa$ And b still stands out as an object that best resembles a low-gravity object.

\section{Limits on Additional Companions at Smaller Angular Separations}

Our data do not reveal any additional companions located interior to $\kappa$ And $\mathrm{b}$. To set limits on companions located interior to $\kappa$ And b, we first divided the $5 \sigma$ residual noise profile in the wavelength-collapsed ADI+SDI image by the median stellar flux. We injected model $\mathrm{L} 0 \gamma$ dwarf spectra from the library of Bonnefoy et al. (2014a) and propagated them through ADI and then SDI to determine their signal loss. We performed 10 iterations of forward-modeling and interpolated the results to create a "flat field" to correct our noise profile map. Due to CHARIS's large bandpass and our use of local (subtraction zone) masking, signal loss from SDI was minor $(\lesssim 20 \%)$, and the radially averaged throughput ranged between $59 \%$ and $73 \%$ from $\rho \sim 0$ !" 3 to $1 . \prime 0$.

To translate our limits on broadband contrast to stellar mass, we used the evolutionary models of Baraffe et al. (2003) to predict values for gravity and temperature and then atmosphere models to determine the "broadband" $(J H K)$ flux density for 3-30 $M_{\mathrm{J}}$ substellar objects at these gravities/temperatures at $40 \mathrm{Myr}$. Values ranged from $T_{\text {eff }} \sim 600 \mathrm{~K}, \log (g)=3.5$ to $T_{\text {eff }} \sim 2300 \mathrm{~K}, \log (g)=4.5$. Atmosphere models draw from A. Burrows, using cloud prescriptions that provide good fits to substellar objects covering most of this range: HR $8799 \mathrm{cde}, \beta$ Pic b, and ROXs 42Bb (Currie et al. 2011; Madhusudhan et al. 2011; Currie et al. 2013, T. Currie et al. 2018, in preparation).

Figure 11 displays our contrast curve. The broadband contrast dips just below $10^{-6}$ at wide separations and gradually increases to $10^{-5}$ at $\rho \sim 0$ ". 35-0." 45 . Despite extremely poor field rotation and $\sim 12$ minutes of integration time, our contrasts exterior to 0 ". $35-0$ ". 45 are comparable to those from SCExAO/HiCIAO for HD 36546-a factor of 3 deeper and a factor of 10 better field rotation (Currie et al. 2017b) -as well as Gemini Planet Imager first-light imaging of $\beta$ Pic $b$, which were likewise much deeper than our data (Macintosh et al. 2014). Companions with contrasts and masses at or below those of $\kappa$ And $\mathrm{b}$ would have been detectable down to 0". 3 (15 au). Any companion more massive than $\kappa$ And $\mathrm{b}$ and capable of scattering it to wide separations must lie within 15 au.

\section{The Orbit of $\kappa$ And $b$}

Well-calibrated astrometry for $\kappa$ And $\mathrm{b}$ now spans five years and reveals a clear change in position with time. Orbital solutions derived for objects with low phase coverage are highly sensitive to input priors on different orbital parameters (Kosmo O'Neil et al. 2018). We use two different approaches -OFTI and ExoSOFT (Blunt et al. 2017; Mede \& Brandt 2017) — and adopt different priors to determine plausible orbital properties of the companion. The first investigation of $\kappa$ And b's orbit was carried out by Blunt et al. (2017); our focus is to improve upon these constraints using a longer time baseline to 

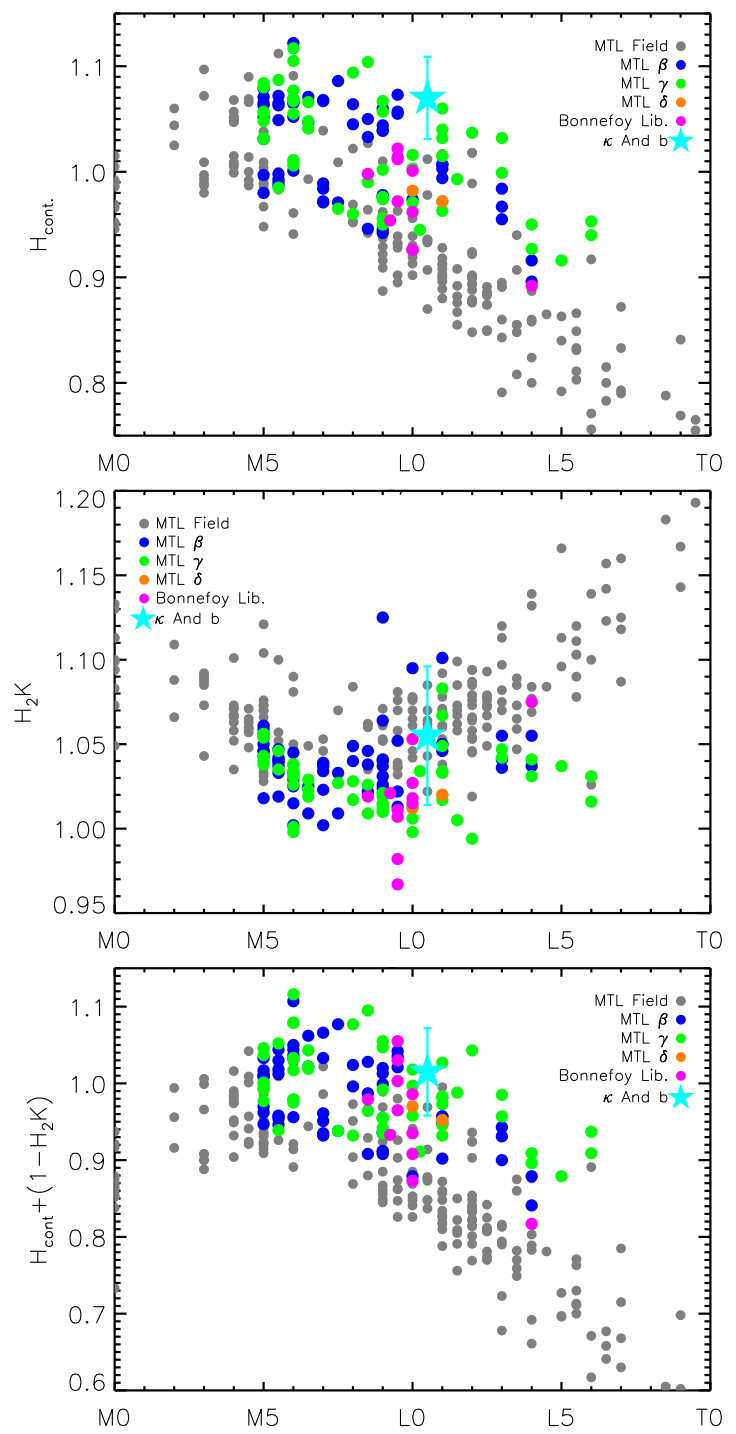

Figure 10. Revised (for CHARIS) $\mathrm{H}$-continuum (top) and $\mathrm{H}_{2} \mathrm{~K}$ (middle) gravity-sensitive spectral indices, and a combined index (bottom) for $\kappa$ And $b$ (cyan star) and the comparison sample. Large values for the $H$-continuum index at a given spectral type suggest low gravity; small values for the $\mathrm{H}_{2} \mathrm{~K}$ index also generally suggest low gravity, albeit less decisively. Uncertainties are shown for $\kappa$ And $\mathrm{b}$; those for the comparison sample are not shown for clarity but are typically of the order of the symbol size.

determine the companion's orbital direction and identify plausible values for its semimajor axis, eccentricity, and orbital inclination.

OFTI uses a Bayesian rejection sampling algorithm to efficiently determine the most plausible orbital parameters. We assume Gaussian priors for the parallax centered on Gaia DR2 catalog values, a uniform prior in stellar mass (2.7-2.9 $\left.M_{\odot}\right)$, and impose a log-normal prior in semimajor axis $\left(a^{-1}\right)$. ExoSOFT uses a Markov chain Monte Carlo approach to determine the orbital fit and posterior distributions, and Simulated Annealing to find reasonable starting positions for the Markov chain and to tune step sizes. We assume a Jeffrey's prior for the semimajor axis $\left(a^{-1} / \ln \left(a_{\max } / a_{\min }\right)\right)$, which gives equal prior probability for the semimajor axis for each decade of parameter space explored. Our astrometric errors conservatively consider the intrinsic $\mathrm{S} / \mathrm{N}$ of the detection, uncertainties in image registration, uncertainties due to self-subtraction/ annealing, and absolute astrometric calibration.

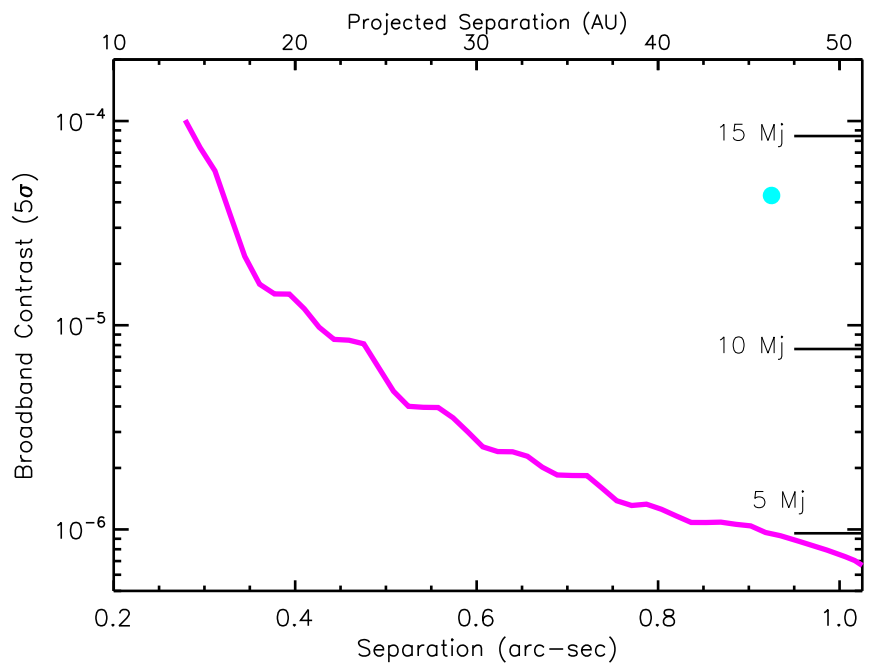

Figure 11. The $5 \sigma$ broadband contrast curve for $\kappa$ And data reduced using ADI + SDI. A cyan circle identifies the position and contrast of $\kappa$ And b; horizontal bars denote the contrast for substellar objects of various masses.

Figure 12 shows orbital fits using OFTI and ExoSOFT and Table 3 lists the median value for orbital parameters and their $68 \%$ confidence intervals. Both approaches determine that $\kappa$ And $b$ orbits clockwise on the plane of the sky, and that its orbit likely has a semimajor axis substantially larger than its projected separation (e.g., 76.5-19.8 au at the $68 \%$ confidence interval for OFTI) and is highly eccentric (e.g., $e \sim 0.69_{-0.10}^{+0.14}$ for ExoSOFT), although astrometric offsets from different epochs can in principle mimic a non-zero eccentricity. OFTI finds a wide range of acceptable orbital inclinations$119^{\circ} .6-157^{\circ} .4\left(111^{\circ} .1-171^{\circ} .5\right.$ within the $95 \%$ confidence interval)-meaning that $\kappa$ And b's orbit is likely inclined $\sim 30^{\circ}-70^{\circ}$ from face-on: a subset of these solutions could imply that the companion's orbital plane is aligned with that of the star $\left(i_{\mathrm{eq}} \sim 60^{\circ}\right)$. ExoSOFT finds slightly lower inclinations although orbits aligned with the star's rotational axis lie within the $95 \%$ confidence interval.

\section{Discussion}

\subsection{New Constraints on the Atmosphere and Orbit of $\kappa$ And $b$}

Our study clarifies the atmospheric and orbital properties of $\kappa$ And $\mathrm{b}$, summarized in Table 4 . Previous studies analyzing broadband photometry and P1640 spectra (Hinkley et al. 2013; Bonnefoy et al. 2014a) admit a wide range of acceptable spectral types or different answers depending on (a) whether field or low-/intermediate-gravity comparison spectra are used or (b) the wavelength range used for matches with empirical spectra. ${ }^{38}$ Comparing the CHARIS spectra to both optically anchored spectral templates and spectral libraries shows that $\kappa$ And $b$ best resembles a young, low-gravity L0-L1 dwarf (LOL1 $\gamma$ ) such as 2MASS J0141-4633. Its $H$-band spectral shape in particular shows strong evidence for a low surface gravity.

A number of factors may explain why our conclusions about $\kappa$ And b's spectrum show small differences from those presented in Hinkley et al. (2013). Chiefly, the $\mathrm{S} / \mathrm{N}$ of $\kappa$ And b's spectrum

\footnotetext{
38 For example, Bonnefoy et al. (2014a) find that M9-L3 objects can match $\kappa$ And b's photometry; Hinkley et al. (2013) find a best fit with an L4 field dwarf and an intermediate-gravity L1 dwarf $(\mathrm{L} 1 \beta)$.
} 

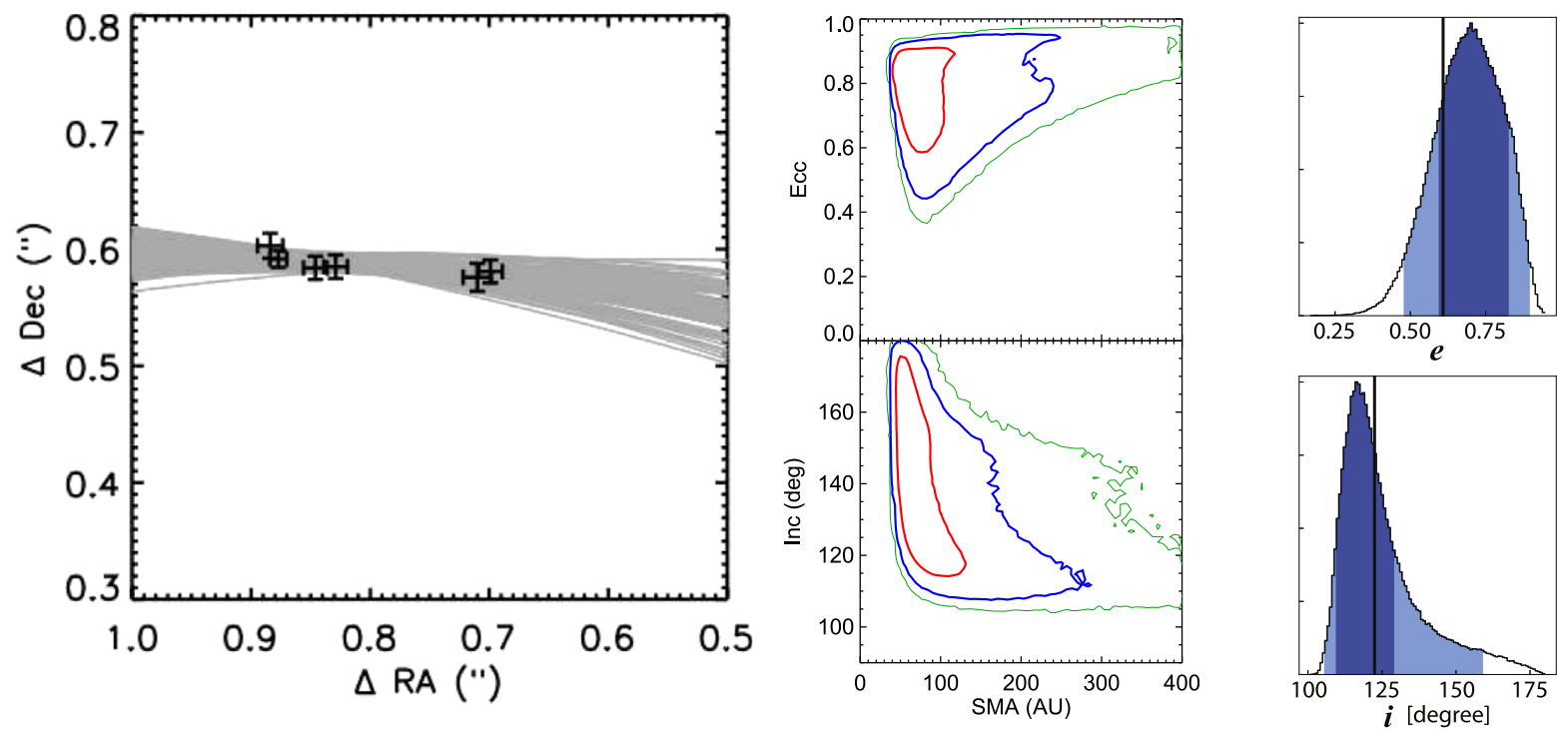

Figure 12. Orbit fitting results using OFTI (Blunt et al. 2017) and ExoSOFT (Mede \& Brandt 2017). Left: the best-fit orbits from OFTI compared to astrometric data. The first two epochs draw from HiCIAO astrometry presented in Carson et al. (2013); the third, fourth, and last epochs are our NIRC 2 astrometry, while the fifth epoch is from CHARIS. The results and the goodness-of-fits from ExoSOFT are comparable. Middle: the 68\% (red) and 95\% (blue) confidence intervals for semimajor axis, inclination, and eccentricity from OFTI. Right: the probability distributions for eccentricity and inclination from ExoSOFT: the dark blue and light blue regions identify the $68 \%$ and $95 \%$ confidence intervals, respectively.

Table 3

Orbit Fitting for $\kappa$ And $\mathrm{b}$

\begin{tabular}{|c|c|c|c|c|c|}
\hline Orbital Element & Unit & $\begin{array}{l}\text { OFTI } \\
\text { Median [68\% C.I.] }\end{array}$ & [95\% C.I.] & $\begin{array}{l}\text { ExoSOFT } \\
\text { Median [68\% C.I.] }\end{array}$ & [95\% C.I.] \\
\hline$a$ & au & $76.5[56.7,128.2]$ & {$[47.2,286.6]$} & $99.0[53.7,126.6]$ & {$[45.1,216.1]$} \\
\hline$P$ & $\mathrm{yr}$ & $399.9[254.9,868.1]$ & {$[193.3,2899.5]$} & $588.8[214.1,825.9]$ & {$[169.0,1868.8]$} \\
\hline$i$ & deg & $136.2[119.6,157.4]$ & {$[111.1,171.5]$} & $121.2[109.2,129.2]$ & {$[105.5,158.7]$} \\
\hline$\omega$ & deg & $126.5[49.1,161.0]$ & {$[3.6,176.7]$} & $129.5[95.7,157.2]$ & {$[71.1,195.5]$} \\
\hline$\Omega$ & deg & $75.9[54.1,100.5]$ & {$[15.4,162.1]$} & $75.7[64.1,87.0]$ & {$[31.1,113.4]$} \\
\hline
\end{tabular}

Note. Orbits are fit to the four new NIRC2 and CHARIS astrometric points plus two HiCIAO epochs listed in Carson et al. (2013).

is substantially higher $\left(\mathrm{S} / \mathrm{N}_{\text {med.,CHARIS }} \sim 20.5\right.$ versus 5 for P1640), in large part owing to SCExAO's extremely highfidelity AO correction, resulting in a deep raw contrast. This allowed us to extract a higher-fidelity spectrum and more clearly identify which spectral templates and empirical spectra match $\kappa$ And $\mathrm{b}$. Furthermore, calibrating the $\kappa$ And $\mathrm{b}$ spectrum from P1640 data is arguably more challenging since it relies on forward-modeling data reduced using SDI only (see Pueyo 2016). The slightly wider, redder bandpass (1.1-2.4 $\mu \mathrm{m}$ versus $0.9-1.8 \mu \mathrm{m}$ ) also probes more of $\kappa$ And b's spectral energy distribution, also aiding the identification of the companion's best-fit spectral properties. Appendix B identifies an additional possible source of differences from the template spectrum used for spectrophotometric calibration.

While Carson et al. (2013) demonstrated that $\kappa$ And b is a bound companion, their short ( $\sim 0.75 \mathrm{yr})$ astrometric baseline precluded a detailed understanding of the companion's orbit, admitting a wide range of parameter space (Blunt et al. 2017). Our astrometry establishes a $5 \mathrm{yr}$ baseline and decisively determined $\kappa$ And b's orbital direction (clockwise). Orbital fits from two separate but complementary codes show that the companion's orbital plane is highly inclined relative to the sky and possibly coplanar with the rotation axis of the star. Its eccentricity is likely substantial. The semimajor axis of $\kappa$ And $\mathrm{b}$ suggests that the companion may orbit at a significantly wider separation than previously thought. The companion's orbitincluding inclination and semimajor axis - can be better clarified by including new astrometric measurements and determining solutions assuming observable-based priors (Kosmo O'Neil et al. 2018).

\section{2. $\kappa$ And $b$ in Context: Constraints/Limits on Temperature, Age, Gravity, Mass, and Formation}

While we reserve a detailed atmospheric modeling analysis of $\kappa$ And $\mathrm{b}$ for a future publication, we can use empirical comparisons to now quantitatively limit its temperature, revisit its age, and estimate its surface gravity and mass. Combining these results with new information on $\kappa$ And b's orbit allows us to revisit a discussion of its plausible formation mechanisms.

Temperature. A subset of the substellar objects whose spectra best fit $\kappa$ And $\mathrm{b}$ have a temperature derived from atmospheric modeling (Bonnefoy et al. 2014b; Faherty et al. 2016). Conveniently, the best-fitting object-2MASS J01414633 - was analyzed in Bonnefoy et al. (2014a) using models incorporating cloud/atmospheric dust prescriptions that accurately reproduce young, early L-dwarf spectrophotometry over 
Table 4

Properties of the $\kappa$ And System

\begin{tabular}{|c|c|c|c|}
\hline Parameter & $\kappa$ And $\mathrm{A}$ & $\kappa$ And $\mathrm{b}$ & Reference \\
\hline \multicolumn{4}{|l|}{ Object } \\
\hline \multicolumn{4}{|l|}{ Properties } \\
\hline$d(\mathrm{pc})$ & $50.0 \pm 0.1$ & $\ldots$ & 1 \\
\hline Age (Myr) & $47_{-40}^{+27}$ & $\approx 40_{-19}^{+34} ?$ & 2,6 \\
\hline Mass & $2.8 M_{\odot}$ & $\approx 13_{-2}^{+12} M_{\mathrm{J}} ?$ & 2,6 \\
\hline $\log \left(L / L_{\odot}\right)$ & $1.80_{-0.04}^{+1.7}$ & $-3.81 \pm 0.05$ & 2,6 \\
\hline Spectral type & B9IV/B9V & $\mathrm{L} 0-\mathrm{L} 1 \gamma$ & $2,5,6$ \\
\hline$T_{\text {eff }}(\mathrm{K})$ & $11327_{-44}^{+421}$ & $1700-2000$ & 2,6 \\
\hline $\log (g)(\operatorname{dex})$ & $4.174_{-0.012}^{+0.019}$ & $\approx 4.0-4.5 ?$ & 2,4 \\
\hline \multicolumn{4}{|l|}{ Photometry } \\
\hline$J$ (mag) & $4.26 \pm 0.04$ & $15.84 \pm 0.09$ & 2 \\
\hline$H$ (mag) & $4.31 \pm 0.05$ & $15.01 \pm 0.07$ & 2 \\
\hline$K_{\mathrm{s}}(\mathrm{mag})$ & $4.32 \pm 0.05$ & $14.37 \pm 0.07$ & 2 \\
\hline$L^{\prime}(\mathrm{mag})$ & $4.32 \pm 0.05$ & $13.12 \pm 0.1$ & 3,4 \\
\hline NB_4.05 (mag) & $4.32 \pm 0.05$ & $13.0 \pm 0.2$ & 4 \\
\hline$M^{\prime}(\mathrm{mag})$ & $4.30 \pm 0.06$ & $13.3 \pm 0.3$ & 4 \\
\hline \multicolumn{4}{|l|}{ Astrometry } \\
\hline UT Date & Data Source & {$[\mathrm{E}, \mathrm{N}](\operatorname{arcsec})$} & \\
\hline 20120101 & $\begin{array}{l}\text { AO188/ } \\
\text { HiCIAO }\end{array}$ & $\begin{array}{l}{[0.884 \pm 0.010} \\
0.603 \pm 0.011]\end{array}$ & 3 \\
\hline 20120708 & $\begin{array}{l}\text { AO188/ } \\
\text { HiCIAO }\end{array}$ & $\begin{array}{c}{[0.877 \pm 0.007} \\
0.592 \pm 0.007]\end{array}$ & 3 \\
\hline 20121103 & Keck/NIRC2 & $\begin{array}{c}{[0.846 \pm 0.010} \\
0.584 \pm 0.010]\end{array}$ & 2 \\
\hline 20130818 & Keck/NIRC2 & $\begin{array}{c}{[0.829 \pm 0.010} \\
0.585 \pm 0.010]\end{array}$ & 2 \\
\hline 20170905 & $\begin{array}{c}\text { SCExAO/ } \\
\text { CHARIS }\end{array}$ & $\begin{array}{c}{[0.710 \pm 0.012} \\
0.576 \pm 0.012]\end{array}$ & 2 \\
\hline 20171209 & Keck/NIRC2 & $\begin{array}{c}{[0.699 \pm 0.010} \\
0.581 \pm 0.010]\end{array}$ & 2 \\
\hline
\end{tabular}

Note. References: (1) Gaia Collaboration, (2) this work, (3) Carson et al. (2013), (4) Bonnefoy et al. (2014a), (5) Hinkley et al. (2013), (6) Jones et al. (2016). We conservatively assign a positional uncertainty in each coordinate to account for the difference between the apparent and actual positions of the star underneath the coronagraph spot (NIRC2, $\sim 0.25$ pixels) or from a polynomial fit to the apparent centroid positions derived from satellite spots (CHARIS, $\sim 0.25$ pixels), uncertainties in the north position angle and pixel scale (larger for CHARIS), the intrinsic S/N (both), uncertainties in the parallactic angle as recorded in the first header (primarily NIRC2), and uncertainties in the astrometry due to self-subtraction/annealing (both, larger for NIRC2). The age, gravity, and mass are not directly measured, so we denote their estimates with "?."

1-5 $\mu \mathrm{m}$ (Burrows et al. 2006; Daemgen et al. 2017, T. Currie et al. 2018 in preparation). Bonnefoy et al. (2014a) derive $T_{\text {eff }}=1800_{-100}^{+200} \mathrm{~K}$. While models utilized to constrain temperature in Faherty et al. (2016) were limiting cases that more poorly fit young, early L dwarfs, the derived temperature estimate for 2MASS J0141-4633 using these models is consistent $(1899 \mathrm{~K} \pm 123 \mathrm{~K})$. Temperatures for 2MASS J0120-5200, 2MASS J0241-5511, and 2MASS J2322-6151B (all $\mathrm{L} 1 \gamma$ ) are slightly lower, as expected, and consistent with the range of $\mathrm{L} 0-\mathrm{L} 1 \gamma$ temperatures listed in Gonzales et al. (2018). Separately, temperatures for the closest-fitting field spectral type (L3) have a comparable range (1800-1900 K; Stephens et al. 2009). Taken together, we estimate a temperature of $1700-2000 \mathrm{~K}$ for $\kappa$ And $\mathrm{b}$.
Age. While a qualitative assessment of "low gravity" generally means "young," the mapping onto age may not be decisive. Specifically, it is not clear yet how systematically different substellar objects are in gravity class from $\sim 10$ Myr to $40 \mathrm{Myr}$ to $100 \mathrm{Myr}$, etc., and population studies may identify some overlap. ${ }^{39}$ Nevertheless, we can use properties of the best-fitting substellar objects coupled with system kinematics and interferometric measurements of the primary to determine whether multiple lines of evidence are consistent with the same likely age of the $\kappa$ And system.

According to the Bayesian analysis tool for identifying movinggroup members, Banyan- $\Sigma$ (Gagne et al. 2018b), four of the seven objects in Table 2 are bona fide, decisive members of Tuc-Hor (>99.7\% membership probability), which has an Li-depletion age of $40_{-19}^{+5}$ Myr (Kraus et al. 2014). A fifth is a "likely" member of Tuc-Hor (53\% probability) and sixth a possible member (25\% probability). The other is a previously identified candidate member of AB Dor (130-200 Myr) (Bell et al. 2015), where previous versions of Banyan (e.g., Banyan-II) estimated a far higher membership probability than does Banyan- $\Sigma$. Tuc-Hor is comparable in age to the Columba association $(t \approx 30-40 \mathrm{Myr}$; Zuckerman et al. 2011; Bell et al. 2015), because the two groups' pre-main sequences (luminosity versus temperature) are nearly identical (Bell et al. 2015). While $\kappa$ And b's proposed membership in Columba is highly suspect (Hinkley et al. 2013), using new Gaia DR2 astrometry Banyan- $\Sigma$ still suggests that it is a possible member (20\% probability). ${ }^{40}$

Thus, regardless of whether $\kappa$ Andromedae actually is a member of Columba, properties of both the primary and companion are consistent with what a system coeval with Columba should look like. Considering all lines of evidence together, we favor an age of $40_{-19}^{+34} \mathrm{Myr}$, where the upper and lower bounds are equated with the upper bound on age for the primary and the lower bound for most best-fitting comparison spectra, respectively. ${ }^{41}$

Gravity. While there are few direct anchors for surface gravity for young substellar objects (see Stassun et al. 2006, 2007; Canty et al. 2013, T. Currie et al. 2018, in preparation), atmosphere/substellar evolution models can help identify plausible values for $\kappa$ And $b$. Although a small subset of best-fit models that reproduced the spectrum of 2MASS J0141-4633 in Bonnefoy et al. (2014b) had high surface gravities expected for field objects $(\log (g) \sim 5-5.5)$, most had $\log (g)=4.0 \pm 0.5$. Using the evolutionary models of Baraffe et al. (2003), this object, siblings in Tuc-Hor, and slightly younger (20 Myr old) ones are predicted to have surface gravities of the order of $\log (g) \sim 4.1-4.2$, while those of comparable temperature near our preferred upper age limit of

\footnotetext{
39 For instance, while all good-fitting Tuc-Hor members are L0-L1 $\gamma$, some L0-L1 objects in much older associations can also have a $\gamma$ designation (e.g., AB Dor candidate member 2MASS J2325-0259). AB Dor includes likely members with both intermediate and low gravities at a given spectral type (Allers \& Liu 2013).

40 Furthermore, the system's kinematics are identical to that of HR 8799 (50\% membership probability) within errors and its space position is similar. Banyan$\Sigma$ also does not consider ancillary information indicating that a particular system is young (e.g., spectral properties) $-\kappa$ And is clearly not a Gyr-old system - and new astrometry obtained with alternative kinematics codes may obtain different results (e.g., Dupuy et al. 2018).

41 Taken at face value, this result appears to contradict that obtained by Hinkley et al. (2013), who find that $\kappa$ And is likely at least $200 \mathrm{Myr}$ old. However, as clearly stated in Hinkley et al., a much younger age is possible if the primary is a fast rotator viewed pole-on, which is exactly what was found in Jones et al. (2016). Thus, our two studies yield consistent answers on the system's age.
} 
$\sim 74$ Myr should have $\log (g) \sim 4.5$. Surface gravities of $\log$ $(g) \sim 4-4.5$ are therefore supported by a joint consideration of detailed atmosphere modeling of best-fitting spectra and predictions from evolutionary models covering $\kappa$ And's most plausible age range so far.

Mass. Armed with a revised estimate for $\kappa$ And b's spectral type, photometry, and the system's distance, we calculate a bolometric luminosity of $\log \left(L / L_{\odot}\right)=-3.81 \pm 0.06$ using the bolometric correction obtained by Todorov et al. (2010) for 2MASS J0141-4633. ${ }^{42}$ Luminosities for the best-fitting L dwarfs in Tuc-Hor are comparable to that of $\kappa$ And b or slightly higher by $0.25 \mathrm{dex}(-3.55$ to -3.8$)$. As their implied masses are $12-15 M_{\mathrm{J}}$, if $\kappa$ And is coeval with Tuc-Hor then $\kappa$ And $b$ is likely lower in mass. Considering the full range of favored system ages, $\kappa$ And b's estimated mass is $13_{-2}^{+12} M_{\mathrm{J}}$ and the companion-to-primary mass ratio is $q \sim 0.005_{-0.001}^{+0.005}$.

Formation. Our results provide new information helpful for assessing how $\kappa$ And $\mathrm{b}$ relates to bona fide planets detected by both indirect techniques and direct imaging and to low-mass brown dwarfs. While the companion's mass is near or may even exceed the deuterium-burning limit, the utility and physical basis of this IAU criterion or any other hard upper limit on mass for a "planet" is unclear (Luhman 2008). ${ }^{43}$ Alternative criteria focusing on the demographics of imaged companions-mass ratio and separation-may more clearly distinguish planets from brown dwarf companions (Kratter et al. 2010; Currie et al. 2011).

While the plausible mass ratios of $\kappa$ And $\mathrm{b}$ are intermediate between those of HR 8799 cde $\left(q \sim 4.5 \times 10^{-3}\right)$ and ROXs $42 \mathrm{Bb}\left(q \sim 9 \times 10^{-3}\right)$, its orbital separation is likely larger than (almost) all HR 8799 planets, more comparable to HIP $65426 \mathrm{~b}$ and ROXs 42Bb (90-150 au; Chauvin et al. 2017; Currie et al. $2014 b$ ). Similar to $\kappa$ And b, no additional companions have been found at smaller separations around HIP 65426 or ROXs 42B (Bryan et al. 2016; Chauvin et al. 2017). Although core accretion struggles to form massive companions in situ beyond 50-100 au, disk instability may yet be a viable mechanism to account for $\kappa$ And b, HIP 65426 b, and ROXs 42Bb (e.g., Rafikov 2005). At least some protoplanetary disks contain a significant amount of mass at separations of 50-150 au scale that could be (and perhaps have been) converted into massive companions via gravitational instability (e.g., Andrews \& Williams 2007; Isella et al. 2016), although direct-imaging surveys show that superjovian-mass planets at these separations are rare (Nielsen et al. 2013; Brandt et al. 2014; Galicher et al. 2016).

\subsection{Future Studies of $\kappa$ And $b$}

Follow-up low-resolution CHARIS spectroscopy in individual passbands $(J / H / K ; R \sim 80)$ could better clarify $\kappa$ And b's atmospheric properties. Gravity-sensitive indices $H$-cont and $\mathrm{H}_{2} \mathrm{~K}$ approximated in this work could be more reliably determined; $J$-band potassium lines $\left(K_{I}\right)$ could provide a third assessment of the companion's gravity (Allers \& Liu 2013). An improved census of substellar objects with ages at or just

\footnotetext{
${ }^{42}$ Using the K-correction from Golimowski et al. (2004) for the best-fit field spectral type (L3) yields very similar results, consistent within errors $\left(\log \left(L / L_{\odot}\right) \sim-3.79\right)$.

43 For example, the quadruple system 2MASS J0441+2301 (Todorov et al. 2010; Bowler \& Hillenbrand 2015) includes two low-mass companions $\left(M \sim 10,20 M_{\mathrm{J}}\right)$, suggesting that binary stars formed from molecular cloud fragmentation could still satisfy the IAU definition of a "planet."
}

greater than that of Columba/Tuc-Hor (40-100 Myr) aided by the identification of new moving groups (e.g., Gagne et al. 2018a) could better establish a context for $\kappa$ And $b$ and how its spectrum compares to the full range of objects with very low, low, or intermediate gravity. Ground-based broadband photometry can bracket CHARIS's coverage and also better probe evidence for clouds and small atmospheric dust, while more precisely constraining the companion's temperature (Currie et al. 2011, 2013; Daemgen et al. 2017). Thermal infrared observations with the James Webb Space Telescope could reveal and help begin to quantify the abundance of $\mathrm{CO}, \mathrm{CH}_{4}$, and $\mathrm{CO}_{2}$ (Beichman \& Green 2018).

Higher-resolution $(R \sim 3000)$ integral field spectroscopy of $\kappa$ And $\mathrm{b}$ achievable with Keck/OSIRIS and later on the Thirty Meter Telescope with IRIS will provide a significant advance in understanding $\kappa$ And b's gravity, clouds, chemistry, and perhaps formation (Larkin et al. 2006, 2016; Wright et al. 2014). OSIRIS and IRIS spectra can measure narrow gravitysensitive lines of iron and sodium (Allers \& Liu 2013). Fitting these spectra with sophisticated forward models or analyzing their atmospheric retrievals should also yield estimates for $\mathrm{CO}$, $\mathrm{H}_{2} \mathrm{O}, \mathrm{CH}_{4}$, and perhaps $\mathrm{NH}_{3}$ abundances from resolved molecular line emission (Barman et al. 2015; Todorov et al. 2016). The carbon-to-oxygen ratio derived from these abundance estimates may provide insights into the formation environment of $\kappa$ And $\mathrm{b}$ and perhaps identification with other directly imaged planets (e.g., Barman et al. 2015).

The anonymous referee provided helpful comments, which improved the quality of this paper. Eric Mamajek, Jonathan Gagne, Sasha Hinkley, Nienke van der Marel, Maxwell Service, and Jessica Lu also provided helpful suggestions and comments on earlier manuscript drafts. We thank Adam Burrows for providing atmosphere models and Sasha Hinkley for sharing the P1640 spectrum for $\kappa$ And $b$. We thank Randy Campbell, Carlos Alvarez, Jasmin Silva, Mitchell Rudisel, and Kevianna Adams for assistance with our Keck observations. We wish to emphasize the pivotal cultural role and reverence that the summit of Maunakea has always had within the indigenous Hawaiian community. We are most fortunate to have the privilege to conduct scientific observations from this mountain and thus acknowledge the responsibility to use telescope time on Maunakea wisely. This research has made use of the Keck Observatory Archive (KOA), which is operated by the W. M. Keck Observatory and the NASA Exoplanet Science Institute (NExScI), under contract with the National Aeronautics and Space Administration. T.C. is supported by a NASA Senior Postdoctoral Fellowship. S.B. is supported by an NSF Graduate Research Fellowship. J.C. received support from the U.S. Fulbright program and from SC Space Grant.

\section{Appendix A CHARIS Astrometric Calibration}

While precise astrometric calibration is ongoing, in this paper we present a preliminary calibration tied to Keck/NIRC2 based on observations in 2017 July, September, and December of the HD 1160 system. HD 1160 has two wide (sub)stellar companions (Nielsen et al. 2012), one of which (HD $1160 \mathrm{~B}$ ) is near the edge of the CHARIS field of view at $\rho \approx 0$ ". 8 . At a projected separation of $r \sim 80 \mathrm{au}$, the low-mass companion HD 1160 B should not experience significant orbital motion 

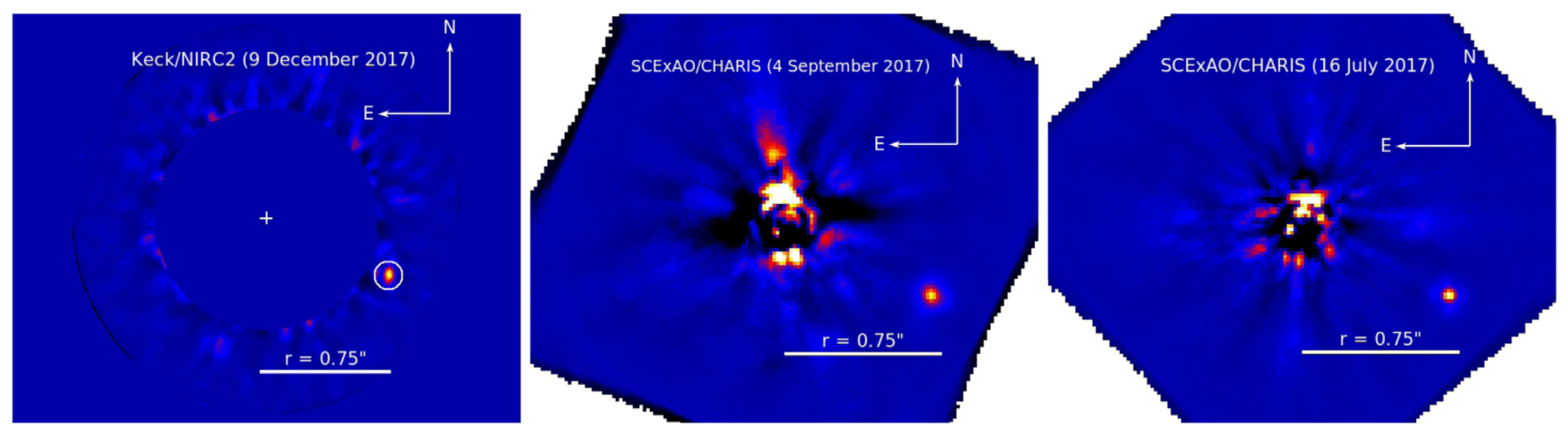

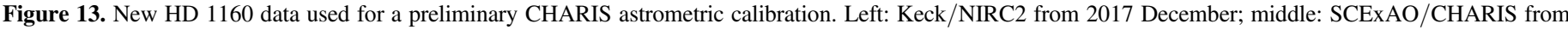

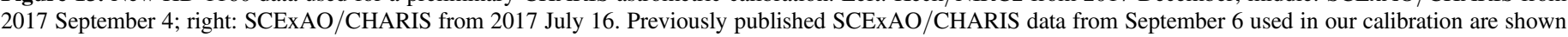
in Currie et al. (2018).

Table 5

Preliminary SCExAO/CHARIS Astrometric Calibration

\begin{tabular}{|c|c|c|c|c|c|}
\hline Telescope/Instrument (Coronagraph) & UT Date & $\rho_{\text {nominal }}(\operatorname{arcsec})$ & $\mathrm{PA}_{\text {nominal }}(\mathrm{deg})$ & $\rho_{\text {corr }}(\operatorname{arcsec})$ & $\mathrm{PA}_{\text {corr }}(\mathrm{deg})$ \\
\hline Keck/NIRC2 (Lyot) & 2017 Dec 9 & $0.784 \pm 0.006$ & $244.93 \pm 0.25$ & $\cdots$ & $\cdots$ \\
\hline SCExAO/CHARIS (Lyot) & 2017 Sep 6 & $0.797 \pm 0.004$ & $242.85 \pm 0.15$ & $0.785 \pm 0.008$ & $245.05 \pm 0.27$ \\
\hline SCExAO/CHARIS (SPC) & 2017 Sep 6 & $0.796 \pm 0.004$ & $242.67 \pm 0.13$ & $0.784 \pm 0.008$ & $244.87 \pm 0.26$ \\
\hline SCExAO/CHARIS (Lyot) & 2017 Sep 4 & $0.796 \pm 0.005$ & $242.60 \pm 0.30$ & $0.784 \pm 0.009$ & $244.80 \pm 0.37$ \\
\hline SCExAO/CHARIS (Lyot) & 2017 Jul 16 & $0.796 \pm 0.004$ & $242.74 \pm 0.15$ & $0.784 \pm 0.008$ & $244.94 \pm 0.27$ \\
\hline
\end{tabular}

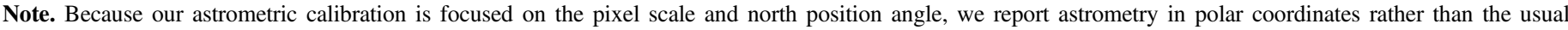

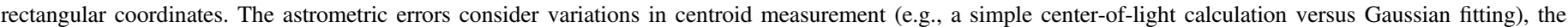
intrinsic S/N of the detection, and (for the CHARIS corrected astrometry) uncertainties in the absolute pixel scale and true north calibration.

(Nielsen et al. 2012; Garcia et al. 2017). Specifically, using the orbital fits from Blunt et al. (2017), the separation and position angle for HD $1160 \mathrm{~B}$ are expected to change by $\Delta \rho \sim$ -0.27 mas \pm 0.36 mas, $\Delta \mathrm{PA} \sim 0.026 \pm 0.01$ between 2017 September and December and $\Delta \rho \sim-0.41$ mas \pm 0.54 mas, $\Delta \mathrm{PA} \sim 0.040 \pm 0.014$ between 2017 July and December. At the separation of HD $1160 \mathrm{~B}$ a change in position angle of 0.04 is no greater than $\sim 5 \%$ of a NIRC2/CHARIS pixel: effectively HD $1160 \mathrm{~B}$ is stationary over this timeframe.

Keck/NIRC2 is precisely calibrated, with an uncertainty in north position angle of 0.02 and an astrometric uncertainty after correction for distortion of 0.5 mas (Service et al. 2016). Thus, we pinned the SCExAO/CHARIS astrometry for HD $1160 \mathrm{~B}$ to that for Keck/NIRC2 to calibrate CHARIS's pixel scale and north position angle offset. This strategy follows that of the Gemini Planet Imager (GPI) campaign team in using contemporaneous GPI and Keck/NIRC2 imaging of HR 8799 to fine-tune GPI's astrometry (Konopacky et al. 2014).

Keck/NIRC2 $K$-band data for HD 1160 were obtained on UT 2017 December 9, immediately after $\kappa$ And, using the 0 ". 6 diameter partially transmissive coronagraphic spot. Images consist of 11 coadded frames covering roughly $5^{\circ}$ in parallactic angle motion. Basic NIRC2 data reduction procedures-flatfielding, dark subtraction, bad pixel mitigation, (post-rebuild) distortion correction, and image registration-follow the pipeline from Currie et al. (2011) that was previously used to process ground-based broadband data. HD $1160 \mathrm{~B}$ was visible in the raw data; no PSF methods were applied. However, the AO correction was modest and the star was blocked by the coronagraph: we assumed a centroid uncertainty of 0.25 pixels in both directions. In determining the error budget, we also considered the intrinsic $\mathrm{S} / \mathrm{N}$ of the detection.
The JHK data for HD 1160 from SCExAO/CHARIS data were previously reported in Currie et al. (2018) and taken on 2017 September 6 in two sequences, one with the Lyot coronagraph and another using the shaped-pupil coronagraph with good AO performance. HD $1160 \mathrm{~B}$ is detected at a high significance in both data sets in all individual channels and data cubes, even without PSF subtraction techniques applied ( $\mathrm{S} / \mathrm{N} \sim 100$ in the wavelength-collapsed, sequence-combined image). To the astrometry extracted from these data, we add astrometry determined from 2017 September 4 (obtained under extremely poor conditions) and 2017 July 16 (obtained under excellent conditions). Nominal astrometric errors consider the intrinsic $\mathrm{S} / \mathrm{N}$ and a conservative estimate for the centroid (set to 0.25 pixels).

Table 5 shows our resulting astrometry for HD 1160 B; Figure 13 show images for NIRC2 data and previously unpublished SCExAO/CHARIS data. For the nominal CHARIS astrometric calibration (0." 0164 pixel $^{-1}$ and no north position angle offset), the CHARIS astrometry displays no significant astrometric deviation between data sets but is systematically offset from the Keck/NIRC2 astrometry. Taking uncertainty-weighted average astrometric offset, we obtain a revised pixel scale of 0 !'0162 pixel $^{-1} \pm 0$ !. 0001 pixel $^{-1}$ and a north position angle offset of $-2^{\circ} .20 \pm 0.27$ east of north (i.e., CHARIS data must be rotated an additional $2^{\circ} .2$ counterclockwise to achieve a north-up image).

\section{Appendix B Absolute Spectrophotometric Calibration}

A key challenge with the new generation of coronagraphic extreme AO facilities is absolute spectrophotometric 

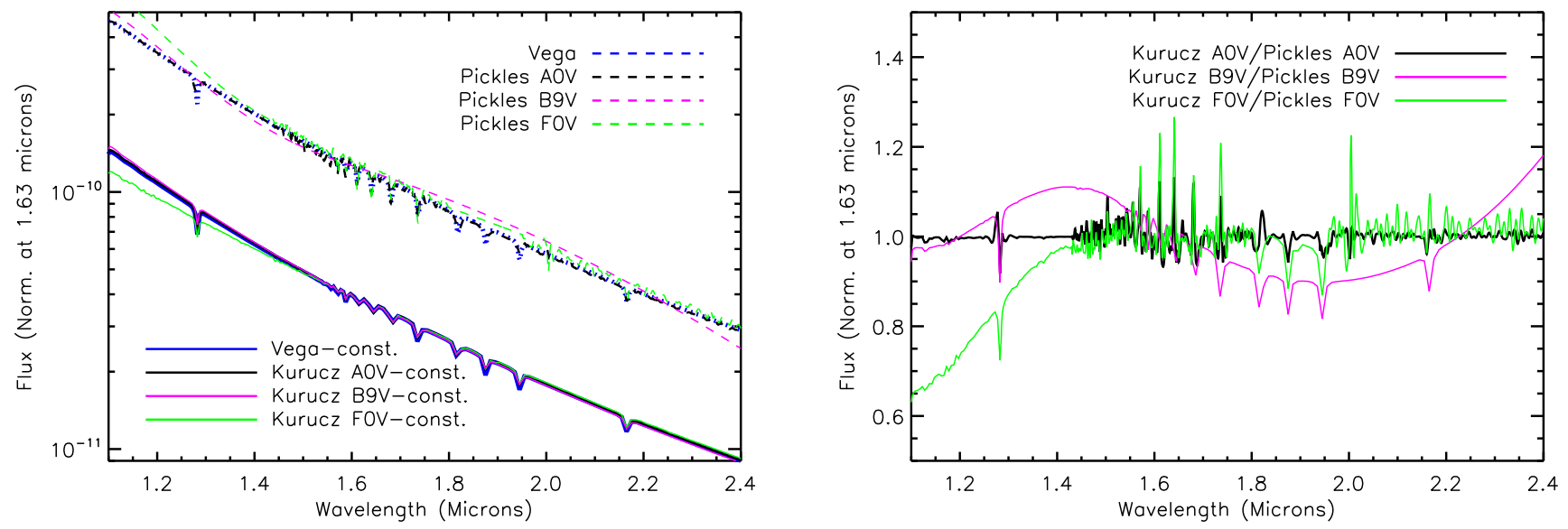

Figure 14. Left: near-infrared spectra from the Pickles library (dashed lines) and from the atmosphere models of Castelli \& Kurucz (2004) (solid lines, offset by a constant). The spectra are normalized for each source at $1.63 \mu \mathrm{m}$ and compared to Vega's spectrum. Despite being of a very similar temperature, the B9V Pickles models exhibit significant offsets that are not predicted by the Kurucz models and are larger than for a photosphere (F0V) whose temperature ( $\sim 7200 \mathrm{~K}$; Currie et al. 2010; Pecaut et al. 2012) is significantly cooler. The Kurucz model spectra are nearly identical except at the shortest wavelengths for the coolest (F0V) model. Right: ratio of the Kurucz to Pickles models for a given spectral type. At B9V, the Pickles models induce errors in spectrophotometric calibration up to $20 \%$.

calibration. Unocculted images of the star are often unavailable and satellite spots of a known attenuation are used to estimate a planet-to-star contrast in each spectral channel. Absolute spectrophotometric calibration is necessary for accurate conclusions about any extracted planet/disk spectrum and requires an accurate model of the intrinsic spectrum of the unresolved target star (or a reference star) (e.g., Currie et al. 2017a).

The Pickles spectral library is the standard source for spectrophotometric calibration in the GPI Data Reduction Pipeline and has been used in direct-imaging discovery and characterization papers (e.g., Macintosh et al. 2015). Importantly, it was used to calibrate P1640 spectra for $\kappa$ And b in Hinkley et al. (2013). However, we opted to use a robust, standard stellar atmosphere model (Castelli \& Kurucz 2004) instead. This is because we identified a potentially serious complication with multiple Pickles library entries at a level important for interpreting low-resolution planet/brown dwarf spectra.

Critically, Pickles (1998) note that near-infrared spectra are present for a few standard spectral types (e.g., A0V) but absent for the vast majority of their library, including the B9V spectral type. For spectral types lacking near-IR spectra, Pickles uses "a smooth energy distribution" extending beyond the reddest available wavelength (typically $1.04 \mu \mathrm{m}$ ) to $5 \mu \mathrm{m}$, such that the integrated broadband photometry in major near-IR passbands matches published values. However, this does not demonstrate that the spectral shape sampled at smaller $\Delta \lambda$ is consistent.

Figure 14 compares B9V and A0V Pickles spectra and counterparts from the Kurucz atmosphere models. The Pickles A0V, Kurucz A0V, and empirical Vega spectrum show strong agreement (left panel). The ratio of the Kurucz A0V to B9V spectrum over the CHARIS passbands is nearly constant, as expected for two objects with similar temperatures and similar exponential terms in their Planck functions (e.g., at $\lambda=[1.25$, $2.15] \mu \mathrm{m}$ this ratio is $[1.27,1.22])$ and a lack of broad molecular absorption features. Thus, we expect a very slowly changing or constant ratio of $\mathrm{A} 0 \mathrm{~V} / \mathrm{B} 9 \mathrm{~V}$ over CHARIS passbands for the Pickles library spectra. However, as clearly shown in the right panel, the $\mathrm{A} 0 \mathrm{~V} / \mathrm{B} 9 \mathrm{~V}$ flux ratio is unexpectedly variable over the CHARIS passbands, deviating by up to $20 \%$ compared to the Kurucz atmosphere models and simple predictions based on pure blackbody emission.

The practical consequence of using the Pickles B9V spectrum with extrapolated near-IR values instead of a stellar atmosphere model would be to suppress $\kappa$ And b's signal at $1.4 \mu \mathrm{m}$ and the red edge of $K$ and increase it at $\sim 1.7-1.8 \mu \mathrm{m}$. These wavelengths overlap with those sampled for gravitysensitive indices. Thus, it is possible that some of our different results for the nature of $\kappa$ And b versus Hinkley et al. (2013) are due to issues with the Pickles B9V spectrum that have only now been highlighted. The choice of a proper stellar library may have important implications for interpreting spectra of substellar objects around other types of stars: for example, a $J$ spectrum extracted for a companion around an F0V star would deviate even more, perhaps leading to a misestimate of the companion's temperature.

\section{Appendix C \\ A Generalized, Robust Forward-Modeling/Spectral Throughput Calibration Using (A-)LOCI}

Powerful advanced least-squares PSF subtraction algorithms such as LOCI, KLIP, and derivatives can bias astrophysical signals, both reducing and changing the spatial distribution of the source intensity, thus affecting both spectophotometry and astrometry (e.g., Marois et al. 2010b; Pueyo et al. 2012). The earliest attempts at correcting for this annealing focused on injecting synthetic point sources at a given separation but different position angles and then processing real data with these sources added in successive iterations to estimate throughput (e.g., Lafrenière et al. 2007). This approach yields a good estimate of the azimuthally averaged point-source throughput suitable for deriving contrast curves; however, it is computationally expensive (e.g., Brandt et al. 2013). Moreover, it is unsuitable for very precise spectrophotometry. This is because algorithm throughput can vary at different angles at a given separation if the intensity of the stellar halo has a high dynamic range (e.g., if it is "clumpy"), since high-signal 
regions contribute more strongly to the residuals that the algorithm seeks to minimize (Marois et al. 2010a).

Forward-modeling provides a way to more accurately recover the intrinsic planet/disk brightness and astrometry/ geometry, where the earliest methods focused on inserting negative copies of a planet PSF into the observing sequence with brightness and position varied until they completely null the observed planet signal (Marois et al. 2010a; Lagrange et al. 2010). With the planet signal entirely removed from the reference library used in these algorithms, PSF-subtracted images containing the planet signal have $100 \%$ throughput (Currie et al. 2014a). While robust, this method is also computationally expensive for IFS data instead of single-band photometry (i.e., the runtime is $n_{\text {channels }}$ longer) or if the intensity distribution of the signal is unknown (e.g., a disk of some morphology) (Pueyo 2016). To circumvent this problem, forward-modeling can be carried out in a more predictive fashion, where coefficients (for LOCI and derivatives) or Karhunen-Loève modes (for KLIP) used for PSF subtraction on science data are applied to empty images/data cubes containing only a synthetic planet or disk model (Soummer et al. 2012; Esposito et al. 2014; Currie et al. 2015a; Pueyo et al. 2015). However, if the planet/disk signal is contained in the reference library used for PSF subtraction, as is usually the case for ground-based imaging, the signal itself can perturb the KL modes/coefficients (Brandt et al. 2013; Pueyo 2016). For KLIP, Pueyo (2016) developed a robust, generalized solution solving this problem, modeling the planet/disk signal as inducing a small perturbation on the KL modes.

Within the classic LOCI formalism, ${ }^{44}$ Brandt et al. (2013) thus far have developed the advanced approach most similar to that done by Pueyo (2016), efficiently modeling the planet as inducing a small perturbation on LOCI coefficients, $\boldsymbol{\beta}$. Their focus was an efficient and rapid computation of contrast curves for broadband data, not precise spectrophotometry/astrometry. In order to precompute the effective planet PSF inducing this perturbation, they therefore approximated it as a Gaussian profile flanked by two sets of Gaussian profiles at fixed position angle offsets and worked in the limiting case that the planet/ disk intensity in the subtraction zone $I_{i, s}^{\prime}$ (not optimization zone) is far lower than the speckle intensity $I_{i, s}$. Absent this robust approach, several authors have introduced modifications such as local masking, a very large optimization zone, or an aggressive singular value cutoff (large $s v d_{\text {lim }}$ ) to substantially reduce the influence of perturbations on LOCI-like algorithms (e.g., Marois et al. 2010b; Currie et al. 2015a). However, we found that these modifications alone failed to yield a high-S $/ \mathrm{N}$ spectrum for $\kappa$ And $\mathrm{b}$, whose throughput is precisely known.

Here, we develop a generalized forward-modeling solution in the (A-)LOCI formalism complementary to the KLIP forward-modeling approach in Pueyo (2016), adopting the formalism of and leveraging upon advances made by Brandt et al. (2013). In the standard case for LOCI-like algorithms, the set of coefficients $\alpha_{i j}$ applied to reference images $I_{j}$ determined from an optimization region $o$ minimize the subtraction

\footnotetext{
44 Pueyo (2016) does describe how to apply his forward-modeling approach to LOCI but with a different linear algebra formalism than utilized in Lafrenière et al. (2007) and nearly all subsequent LOCI-based works, including Brandt et al. (2013).
}

residuals $\mathcal{R}_{i, o}^{2}$ over pixels $k$ for science image $I_{i}$ :

$$
\mathcal{R}_{i, o}^{2}=\sum_{k}\left(I_{i k, o}-\sum_{j} \alpha_{i j} I_{j k, o}\right)^{2} .
$$

Following Brandt et al. (2013), we can perturb this equation by adding a planet PSF of signal $I^{\prime}$ to each reference image $j$ over pixels $k$, inducing a small perturbation in coefficients $\beta_{i j}$; thus the subtraction residuals are now

$$
\begin{aligned}
\mathcal{R}_{i, o}^{2}= & \sum_{k}\left[I_{i k, o}+I_{i k, o}^{\prime}-\sum_{j}\left(\alpha_{i j} I_{j, o}+\alpha_{i j} I_{j k, o}^{\prime}\right.\right. \\
& \left.\left.+\beta_{i j} I_{j k, o}+\beta_{i j} I_{j k, o}^{\prime}\right)\right]^{2} .
\end{aligned}
$$

Linearizing now around $\beta_{i j}$ instead of $\alpha_{i j}$, we find

$$
\begin{aligned}
\frac{\partial \mathcal{R}_{i, o}^{2}}{\partial \beta_{i l}}= & 0=\sum_{k}\left[( I _ { l k , o } + I _ { l k , o } ^ { \prime } ) \left(I_{i k, o}+I_{i k, o}^{\prime}\right.\right. \\
& \left.\left.-\sum_{j}\left(\alpha_{i j} I_{j k, o}+\alpha_{i j} I_{j k, o}^{\prime}+\beta_{i j} I_{j k, o}+\beta_{i j} I_{j k, o}^{\prime}\right)\right)\right] .
\end{aligned}
$$

Over the optimization zone $o$ (not the subtraction zone) and summed over pixels $k$, we assume that the speckle halo intensity is much larger than that of a planet $\left(I_{i, o} \gg I_{i, o}^{\prime}\right)$ and that the speckle halo subtracted by the reference library weighted by the nominal (A-)LOCI coefficients is zero $\left(I_{i, o}-\sum_{j}\left(\alpha_{i j, o} I_{j, o}\right) \sim 0\right)$. This reduces to a system of linear equations

$$
\sum_{k} I_{l k, o}\left(I_{i k, o}^{\prime}-\sum_{j} \alpha_{i j} I_{j k, o}^{\prime}\right)=\sum_{j} \beta_{i j} \sum_{k} I_{l k, o} I_{j k, o},
$$

which can be solved by matrix inversion as done in Equation (4),

$$
\beta=\left(\boldsymbol{U} \boldsymbol{\Sigma}_{>\text {svd }} \boldsymbol{V}\right)^{-1} \cdot \boldsymbol{b}^{\prime},
$$

where $\boldsymbol{U} \boldsymbol{\Sigma} \boldsymbol{V}(\boldsymbol{A})$ is the same covariance matrix whose array elements are described in Equation (3) and $\boldsymbol{b}^{\prime}$ is the column matrix describing how the effective (partially annealed) planet PSF induces a set of perturbations with coefficients $\beta$ :

$$
\mathrm{b}_{l}^{\prime}=\sum_{k} I_{l k, o}\left(I_{i k, o}^{\prime}-\sum_{j} \alpha_{i j, o} I_{j k, o}^{\prime}\right) .
$$

The residual emission of a planet in frame $i$ within subtraction zone $s$ is then

$$
\mathcal{R}_{i, s}^{\prime}=\sum_{k}\left[I_{i k, s}^{\prime}-\sum_{j}\left(\alpha_{i j, o} I_{j k, s}^{\prime}+\beta_{i j, o} I_{j k, s}+\beta_{i j, o} I_{j k, s}^{\prime}\right)\right] .
$$

Throughout, we use the satellite spots to produce a model planet PSF for each channel. The empty data cubes containing the annealed planet PSF produced from the forward model are then derotated and combined. Their photometry and astrometry are compared to input values to derive throughput for each spectral channel and astrometric offsets. These offsets were then applied to our spectral extraction subroutine to derive a flux-calibrated CHARIS spectrum. To confirm the validity of our forward model, we inserted a negative copy of the extracted, corrected CHARIS spectrum into the real data at its predicted position in each data cube $\boldsymbol{x}-I_{i, \lambda, \text { neg. }}=I_{i, \lambda}-I_{i, \lambda}^{\prime \prime}(\boldsymbol{x})$ - and slightly varied the position/brightness of the spectrum to verify that the forwardmodeled solution is the correct one. 

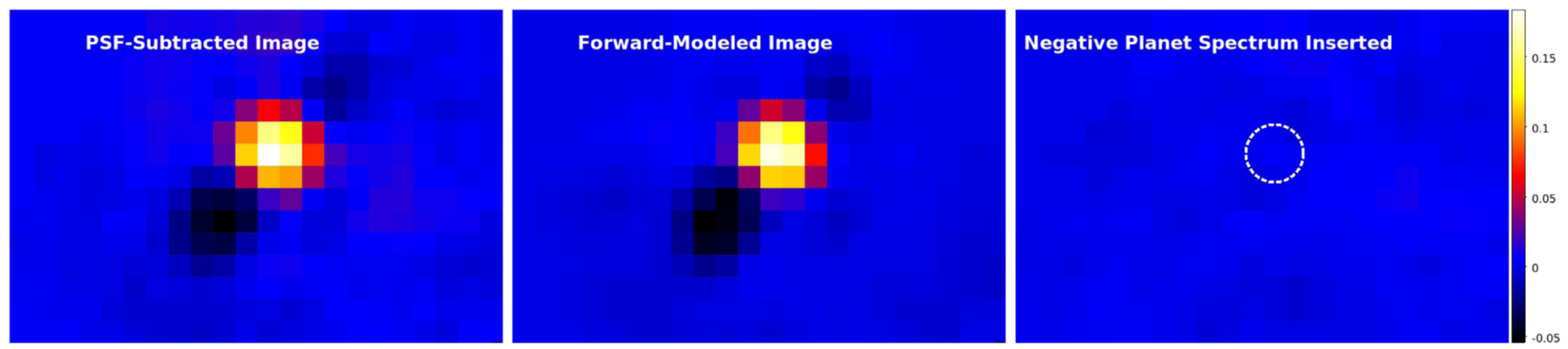

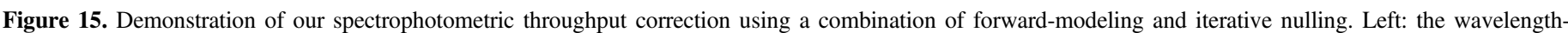

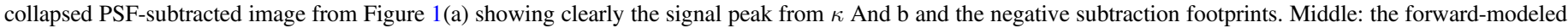

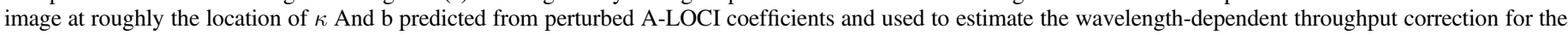

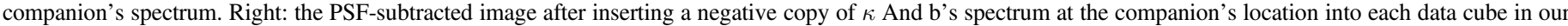

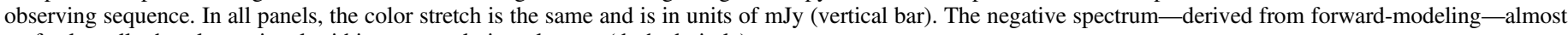
perfectly nulls the planet signal within one resolution element (dashed circle).

Figure 15 illustrates our forward-modeling method, demonstrating that the predicted annealed planet PSF matches the real one and the extracted CHARIS spectrum nulls the planet signal in the sequence-combined data cube. For our $\kappa$ And b data set using algorithm parameters adopted in Section 2, the spectrum throughput ranges between $77 \%$ and $92 \%$; the astrometric offset is $\sim 0.2$ pixels. In classic LOCI, very small optimization zones such as those we adopt combined with poor field rotation can result in low throughput (Lafrenière et al. 2007). Our throughput is high and perturbations of the (A-)LOCI coefficients are low in large part because of local masking (see also Currie et al. 2013). This is because pixels corresponding to the subtraction zone $s$ (ostensibly containing most of the planet signal) are removed from the optimization zone $o$. Since the perturbed coefficients $\boldsymbol{\beta}$ are determined from a system of linear equations considering the optimization zone only, their values are much smaller when local masking is used. Truncating the covariance matrix $\boldsymbol{A}$ also reduces the aggressiveness of the algorithm and potentially the planet's perturbations of (A-)LOCI coefficients.

This approach introduces some key modifications to that first proposed by Brandt et al. (2013) and utilized in the ACORNS pipeline. First, we explicitly calculate the effective planet PSF in each frame $i-I_{i k, o}^{\prime}-\sum_{j} \alpha_{i j, o}$ - since the angular displacements of PSFs in frames $j$ and the coefficients $\alpha_{i j, o}$ are unique for each data set. Second, while we assumed that $I_{i, o} \gg I_{i, o}^{\prime}$ to determine $\boldsymbol{\beta}$, the planet signal over the (typically much smaller) subtraction zone may not always be negligible in each spectral channel. Third, ACORNS (developed for low-Strehl data sets) modeled the planet PSF with a Gaussian intensity distribution, whereas we use an empirical model unique for each data set. Fourth, it explicitly incorporates the distinction between optimization and subtraction zones and incorporates local masking and covariance matrix truncation, although ACORNS can easily be modified to do this as well.

\section{Appendix D}

\section{Extracted SCExAO/CHARIS Spectrum for $\boldsymbol{\kappa}$ And b}

We provide our extracted SCExAO/CHARIS spectrum for $\kappa$ And $\mathrm{b}$ in Table 6 .
Table 6

SCExAO/CHARIS Spectrum in Flux Units (Observed)

\begin{tabular}{lcc}
\hline \hline Wavelength $(\mu \mathrm{m})$ & $F_{\nu}(\mathrm{mJy})$ & $\sigma F_{\nu}(\mathrm{mJy})$ \\
\hline 1.1596 & 0.5910 & 0.0567 \\
1.1997 & 0.6112 & 0.0529 \\
1.2412 & 0.6942 & 0.0498 \\
1.2842 & 0.8349 & 0.0487 \\
1.3286 & 0.7658 & 0.0426 \\
1.3746 & 0.2586 & 0.0403 \\
1.4222 & 0.5371 & 0.0395 \\
1.4714 & 0.5830 & 0.0393 \\
1.5224 & 0.8103 & 0.0397 \\
1.5750 & 0.8248 & 0.0405 \\
1.6296 & 1.1274 & 0.0375 \\
1.6860 & 1.2744 & 0.0351 \\
1.7443 & 1.0190 & 0.0360 \\
1.8047 & 0.8616 & 0.0310 \\
1.8672 & 0.6344 & 0.0508 \\
1.9318 & 0.9750 & 0.0429 \\
1.9987 & 0.8215 & 0.0360 \\
2.0678 & 1.0233 & 0.0338 \\
2.1394 & 1.2442 & 0.0477 \\
2.2135 & 1.3643 & 0.0492 \\
2.2901 & 1.2739 & 0.0590 \\
2.3693 & 1.2030 & 0.0832 \\
\hline
\end{tabular}

Note. Spectra are extracted from our conservative ADI/A-LOCI reduction and corrected for throughput losses. Measurements in regions with non-negligible telluric contamination and/or poor coverage from the template/empirical comparisons ( $\lambda=1.3746,1.4222,1.4714,1.8672,1.9318$, and $1.9987 \mu \mathrm{m})$ were not used in our analysis.

\section{ORCID iDs}

Thayne Currie (i) https://orcid.org/0000-0002-7405-3119 Timothy D. Brandt (i) https://orcid.org/0000-0003-2630-8073 Taichi Uyama (1) https://orcid.org/0000-0002-6879-3030 Sarah Blunt (i) https://orcid.org/0000-0002-3199-2888 Olivier Guyon (1) https://orcid.org/0000-0002-1097-9908 Motohide Tamura (1) https://orcid.org/0000-0002-6510-0681 Christian Marois (i) https://orcid.org/0000-0002-4164-4182 Kyle Mede 느 https://orcid.org/0000-0003-1329-0409 Klaus Hodapp (1) https://orcid.org/0000-0003-0786-2140 Jeffrey Chilcote (1) https://orcid.org/0000-0001-6305-7272 Frantz Martinache (1) https://orcid.org/0000-0003-1180-4138 
Sean Goebel (1) https://orcid.org/0000-0003-3763-2418 Eiji Akiyama (1) https://orcid.org/0000-0002-5082-8880 Ruben Asensio-Torres (i) https://orcid.org/0000-00032990-0726

Markus Janson (10 https://orcid.org/0000-0001-8345-593X

Gillian R. Knapp (ㄱ) https://orcid.org/0000-0002-9259-1164

Jungmi Kwon (1) https://orcid.org/0000-0003-2815-7774

Daehyeon Oh (1) https://orcid.org/0000-0003-2691-804X

Joshua Schlieder (10) https://orcid.org/0000-0001-5347-7062

Michael Sitko (1) https://orcid.org/0000-0003-1799-1755

\section{References}

Allers, K., \& Liu, M. C. 2013, ApJ, 772, 79

Andrews, S. M., \& Williams, J. P. 2007, ApJ, 659, 705

Aritgau, E., Radigan, J., Folkes, S., et al. 2010, ApJL, 718, L38

Baraffe, I., Chabrier, G., Barman, T. S., et al. 2003, A\&A, 402, 701

Barman, T. S., Konopacky, Q., Macintosh, B., \& Marois, C. 2015, ApJ, 804,61

Barman, T. S., Macintosh, B., Konopacky, Q., \& Marois, C. 2011, ApJL, 735, L39

Beichman, C. A., \& Green, T. P 2018, arXiv: 1803.03730

Bejar, V. J. S., Zapatero Osorio, M. R., Pérez-Garrido, A., et al. 2008, ApJL, 673, L185

Bell, C., Mamajek, E. E., \& Naylor, T. 2015, MNRAS, 454, 593

Blunt, S., Nielsen, E., DeRosa, R., et al. 2017, AJ, 153, 229

Bonnefoy, M., Chauvin, G., Lagrange, A.-M., et al. 2014b, A\&A, 562, 127

Bonnefoy, M., Currie, T., Marleau, G.-D., et al. 2014a, A\&A, 562, 111

Bonnefoy, M., Zurlo, A., Baudino, J.-L., et al. 2016, A\&A, 587, 58

Boss, A. 1997, Sci, 276, 1836

Bowler, B. P., \& Hillenbrand, L. A. 2015, ApJL, 811, L30

Brandt, T. D., \& Huang, C. X. 2015, ApJ, 807, 58

Brandt, T. D., McElwain, M., Turner, E. L., et al. 2013, ApJ, 764, 183

Brandt, T. D., McElwain, M., Turner, E. L., et al. 2014, ApJ, 794, 159

Brandt, T. D., Rizzo, M., Groff, T., et al. 2017, JATIS, 3, 8002

Bryan, M., Bowler, B. P., Knutson, H., et al. 2016, ApJ, 827, 100

Burress, R., Hinkley, S., Wahl, M., et al. 2013, in Proc. Third AO4ELT Conf., ed. S. Esposito \& L. Fini, 52, http://ao4elt3.sciencesconf.org/

Burrows, A., Sudarsky, D., \& Hubeny, I. 2006, ApJ, 640, 1063

Canty, J. I., Lucas, P. W., Roche, P. F., \& Pinfield, D. J. 2013, MNRAS, 435,2650

Carson, J., Thalmann, C., Janson, M., et al. 2013, ApJL, 763, L32

Castelli, F., \& Kurucz, R. 2004, arXiv:astro-ph/0405087

Chauvin, G., Desidera, S., Lagrange, A.-M., et al. 2017, A\&A, 605, L9

Chauvin, G., Gratton, R., Bonnefoy, M., et al. 2018, A\&A, 617, A76

Chilcote, J., Pueyo, L., De Rosa, R., et al. 2017, AJ, 153, 182

Cruz, K., Nunez, A., Burgasser, A. J., et al. 2018, AJ, 155, 34

Currie, T., Brittain, S., Grady, C., et al. 2017a, RNAAS, 1, 40

Currie, T., Burrows, A., Girard, J., et al. 2014a, ApJ, 795, 133

Currie, T., Burrows, A., Itoh, Y., et al. 2011, ApJ, 729, 128

Currie, T., Burrows, A., Madhusudhan, N., et al. 2013, ApJ, 776, 15

Currie, T., Cloutier, R., \& Brittain, S. 2015a, ApJL, 814, L7

Currie, T., Daemgen, S., Debes, J., et al. 2014b, ApJL, 780, L30

Currie, T., Debes, J., Rodigas, T., et al. 2012, ApJL, 760, L32

Currie, T., Guyon, O., Tamura, M., et al. 2017b, ApJL, 836, L15

Currie, T., Hernandez, J., Irwin, J., et al. 2010, ApJS, 186, 191

Currie, T., Kasdin, N. J., Groff, T., et al. 2018, PASP, 130, 044505

Currie, T., Lisse, C., Kuchner, M., et al. 2015b, ApJL, 807, L7

Currie, T., Muto, T., Kudo, T., et al. 2014c, ApJL, 796, L30

Cushing, M. C., Marley, M. S., Saumon, D., et al. 2008, ApJ, 678, 1372

Cushing, M. C., Rayner, J., \& Vacca, W. 2005, ApJ, 623, 1115

Daemgen, S., Todorov, K., Silva, J., et al. 2017, A\&A, 601, 65

David, T. J., \& Hillenbrand, L. A. 2015, ApJ, 804, 146

Delorme, P., Gagne, J., Malo, L., et al. 2012, A\&A, 548, 26

Dupuy, T., Liu, M. C., Allers, K. N., et al. 2018, AJ, 156, 57

Esposito, T., Fitzgerald, M., Graham, J., \& Kalas, P. 2014, ApJ, 780, 25

Faherty, J. K., Riedel, A. C., Cruz, K. L., et al. 2016, ApJS, 225, 10

Gagne, J., Faherty, J., Lafreniere, D., et al. 2015, ApJS, 219, 33

Gagne, J., Faherty, J., \& Mamajek, E. 2018a, ApJ, 865, 136
Gagne, J., Lafreniere, D., Duyon, R., et al. 2014, ApJ, 783, 121

Gagne, J., Mamajek, E., Malo, L., et al. 2018b, ApJ, 856, 23

Galicher, R., Marois, C., Macintosh, B., et al. 2016, A\&A, 594, 63

Garcia, E. V., Currie, T., Guyon, O., et al. 2017, ApJ, 834, 162

Goebel, S., Currie, T., Guyon, O., et al. 2018, AJ, in press, arXiv:1810.09458

Golimowski, D. A., Henry, T. J., Krist, J. E., et al. 2004, AJ, 128, 1733

Gonzales, E. C., Faherty, J. K., Gagne, J., et al. 2018, ApJ, 864, 100

Greco, J., \& Brandt, T. D. 2016, ApJ, 833, 134

Groff, T. D., Kasdin, N. J., Limbach, M., et al. 2015, Proc. SPIE, 9605, 96051C

Groff, T. D., Kasdin, N. J., Limbach, M. A., et al. 2014, Proc. SPIE, 9147, 91471W

Hinkley, S., Pueyo, L., Faherty, J. K., et al. 2013, ApJ, 779, 153

Isella, A., Guidi, G., Testi, L., et al. 2016, PhRvL, 117, 251101

Jones, J., White, R. J., Quinn, S., et al. 2016, ApJL, 822, L3

Jovanovic, N., Guyon, O., Martinache, F., et al. 2015b, ApJL, 813, L24

Jovanovic, N., Martinache, F., Guyon, O., et al. 2015a, PASP, 127, 890

Kenyon, S. J., \& Bromley, B. 2009, ApJL, 690, L140

Keppler, M., Benisty, M., Muller, A., et al. 2018, A\&A, 617, A44

Kirkpatrick, J. D., Barman, T. S., Burgasser, A. J., et al. 2006, ApJ, 639, 1120

Knapp, G. R., Leggett, S. K., Fan, X., et al. 2004, AJ, 127, 3553

Konopacky, Q., Thomas, S., Macintosh, B., et al. 2014, Proc. SPIE, 9147, 914784

Kosmo O'Neil , K., Martinez, G., Hees, A., et al. 2018, arXiv:809.05490

Kratter, K., Murray-Clay, R., Youdin, A., et al. 2010, ApJ, 710, 1375

Kraus, A., Shkolnik, E. L., Allers, K., \& Liu, M. C. 2014, AJ, 147, 146

Kuhn, J., Serabyn, E., Lozi, J., et al. 2018, PASP, 130, 985

Kuzuhara, M., Tamura, M., Kudo, T., et al. 2013, ApJ, 774, 11

Lafrenière, D., Marois, C., Duyon, R., et al. 2007, ApJ, 660, 770

Lagrange, A.-M., Bonnefoy, M., Chauvin, G., et al. 2010, Sci, 329, 57

Lambrechts, M., \& Johansen, A. 2012, A\&A, 544, 32

Larkin, J., Barczys, M., Krabbe, A., et al. 2006, Proc. SPIE, 6269, 62691A

Larkin, J., Moore, A. M., Wright, S. A., et al. 2016, Proc. SPIE, 9908, 99081W

Luhman, K. 2008, in ASP Conference Ser. 398, Extreme Solar Systems, ed. D. Fischer et al. (San Francisco, CA: ASP), 357

Macintosh, B., Graham, J., Ingraham, P., et al. 2014, PNAS, 111, 35

Macintosh, B., Graham, J. R., Ingraham, P., et al. 2015, Sci, 350, 64

Madhusudhan, N., Burrows, A., \& Currie, T. 2011, ApJ, 737, 34

Marois, C., Lafreniére, D., Duyon, R., et al. 2006, ApJ, 641, 556

Marois, C., Macintosh, B., Barman, T., et al. 2008, Sci, 322, 1348

Marois, C., Macintosh, B., \& Veran, J.-P. . 2010b, Proc. SPIE, 7736, 52

Marois, C., Zuckerman, B., Konopacky, Q., et al. 2010a, Natur, 468, 1080

Mawet, D., Milli, J., Wahhaj, Z., et al. 2014, ApJ, 792, 97

Mede, K., \& Brandt, T. D. 2017, AJ, 153, 135

Naud, M.-E., Aritgau, E., Malo, L., et al. 2014, ApJ, 787, 5

Nielsen, E., Liu, M. C., Wahhaj, Z., et al. 2012, ApJ, 750, 53

Nielsen, E., Liu, M. C., Wahhaj, Z., et al. 2013, ApJ, 776, 4

Pecaut, M., Mamajek, E., \& Bubar, E. 2012, ApJ, 746, 154

Perrin, M., Maire, J., Ingraham, P., et al. 2014, SPIE, 9147, 3

Peters, M., Groff, T. D., Kasdin, N. J., et al. 2012, Proc. SPIE, 8446, 84462E Pickles, A. 1998, PASP, 110, 863

Pueyo, L. 2016, ApJ, 824, 114

Pueyo, L., Crepp, J. R., Vasisht, G., et al. 2012, ApJS, 199, 6

Pueyo, L., Soummer, R., Hoffman, J., et al. 2015, ApJ, 803, 31

Quanz, S., Meyer, M. R., Kenworthy, M., et al. 2013, ApJL, 766, L1

Rafikov, R. 2005, ApJL, 621, L69

Rajan, A., Rameau, J., De Rosa, R., et al. 2017, AJ, 154, 10

Rameau, J., Chauvin, G., Lagrange, A.-M., et al. 2013, ApJL, 779, L26

Robert, J., Gagne, J., Artigau, E., et al. 2016, ApJ, 830, 144

Savransky, D. 2015, ApJ, 800, 100

Service, M., Lu, J. R., Campbell, R., et al. 2016, PASP, 128, 095004

Slesnick, C., Hillenbrand, L. A., \& Carpenter, J. M. 2004, ApJ, 610, 1045

Soummer, R., Pueyo, L., \& Larkin, J. 2012, ApJL, 755, L28

Stassun, K., Mathieu, R., \& Valenti, J. A. 2006, Natur, 440, 311

Stassun, K., Mathieu, R., \& Valenti, J. A. 2007, ApJ, 664, 1154

Stephens, D., Leggett, S. K., Cushing, M. C., et al. 2009, ApJ, 702, 154

Todorov, K., Line, M., Pineda, J., et al. 2016, ApJ, 823, 14

Todorov, K., Luhman, K. L., \& McLeod, B. 2010, ApJL, 714, L84

Wright, S. A., Larkin, J. E., Moore, A. M., et al. 2014, Proc. SPIE, 9147, $91479 \mathrm{~S}$

Yelda, S., Lu, J. R., Ghez, A., et al. 2010, ApJ, 725, 331

Zuckerman, B., Rhee, J., Song, I., et al. 2011, ApJ, 732, 61 